\title{
Constraining the Inert Doublet Model using Vector Boson Fusion
}

\author{
Daniel Dercks ${ }^{1, \mathrm{a}}$, Tania Robens ${ }^{2, \mathrm{~b}}$ \\ ${ }^{1}$ DESY Hamburg, Notkestrasse 85, 22607 Hamburg, Germany \\ 2 Theoretical Physics Division, Rudjer Boskovic Institute, 10002 Zagreb, Croatia
}

Received: 7 February 2019 / Accepted: 25 October 2019 / Published online: 14 November 2019

(C) The Author(s) 2019

\begin{abstract}
In this work, we use a recast of the Run II search for invisible Higgs decays within Vector Boson Fusion to constrain the parameter space of the Inert Doublet model, a two Higgs doublet model with a dark matter candidate. When including all known theoretical as well as collider constraints, we find that the above can rule out a relatively large part in the $m_{H}, \lambda_{345}$ parameter space, for dark scalar masses $m_{H} \leq 100 \mathrm{GeV}$. Including the latest dark matter constraints, a smaller part of parameter space remains which is solely excluded from the above analysis. We also discuss the sensitivity of monojet searches and multilepton final states from Run II.
\end{abstract}

\section{Introduction}

The Inert Doublet model (IDM) is one of the most straightforward extensions of the Standard Model (SM) [1-3]. It belongs to the class of Two Higgs Doublet Models (2HDM) which contain two $S U(2)$ doublets in the scalar sector. One of these doublets, $\phi_{S}$, has a nonvanishing vacuum expectation value (vev) which is responsible for the spontaneous breaking of electroweak symmetry in the Standard Model while the second scalar doublet $\phi_{D}$ by construction does not acquire such a vev. This second doublet is hence not involved in the spontaneous mass generation in the Standard Model and does not couple to the SM fermions.

Within this model we impose an additional $\mathrm{Z}_{2}$ symmetry, labelled $D$-symmetry, defined via the transformation

$\phi_{D} \rightarrow-\phi_{D}, \phi_{S} \rightarrow \phi_{S}, \mathrm{SM} \rightarrow \mathrm{SM}$,

which should be respected by the Lagrangian and the vacuum.

As electroweak symmetry breaking in this model proceeds completely analogous to the SM without the second doublet,

\footnotetext{
a e-mail: daniel.dercks@ desy.de

b e-mail: trobens@irb.hr
}

$\phi_{S}$ provides the SM-like Higgs particle and is assumed to be even under the $D$ symmetry. The second inert or dark doublet contains two charged and two neutral scalars and as they are odd under the imposed $D$-parity, its lightest neutral component provides a natural candidate for dark matter (DM). It provides a "perfect example" of a WIMP [4-7], and leads to an interesting pattern for the evolution of the Universe, towards the Inert phase as given by the IDM, with one, two or three phase transitions [8]. Furthermore, the IDM can provide a strong first-order phase transition [9-13] as required by the Sakharov conditions to generate a baryon asymmetry of the Universe. After the discovery of a SM-like Higgs particle in 2012, many studies have been performed in the context of the IDM which use Higgs measurements as well as astrophysical observations, see e.g. [14-23]. ${ }^{1}$ In addition, proposals were made how to search for dark scalars at the LHC in leptonic final states $[15,22,28-31]$ and in single or dijet channels [32,33].

Recently, also the important issue of vacuum (meta-) stability in the IDM has been discussed, and it was found that additional, possibly heavy scalars can have a strong impact on it $[19,34-36] .^{2}$

While the model is intriguing per se and in spite of benchmark scenarios for the current LHC run [22,39], it has not yet been studied explicitly by the LHC collaborations. However, recasts of other BSM searches with similar topologies have been presented in the literature, with prominent examples for searches for supersymmetric particles at LEP [40] as well as the first LHC run [20].

In this work, we present a recast of the Run II analyses presented in Ref. [41] by the CMS collaboration which target an invisibly decaying SM-like Higgs boson produced in vector boson fusion (VBF), and Ref. [42] by the ATLAS collaboration which focusses on monojet final states. We reinterpret

\footnotetext{
${ }^{1}$ Recent analyses for models which extend the IDM by an additional singlet have been performed in [24-27].

${ }^{2}$ Similar solutions can be found in a simple singlet extension of the SM Higgs sector, cf. e.g. [37,38] and references therein.
} 
the results of these searches within the IDM by making use of the CheckMATE $[43,44]$ framework.

The regions considered in this work are tested against all currently available theoretical and experimental constraints, with scan procedure and limits as described in Refs. [22,23, 45].

We explore the reach of the above searches for the model's parameter space and identify regions which cannot be excluded by any of the other tested constraints. Finally, we briefly comment on other experimental BSM searches at LHC Run II that could be used as recasts for the IDM and are expected to yield further constraints on its parameter space.

\section{The model}

Imposing symmetry under the $D$-transformation given in Eq. (1), the full scalar potential of the IDM is given by

$$
\begin{aligned}
V\left(\phi_{S}, \phi_{D}\right)= & -\frac{1}{2}\left[m_{11}^{2}\left(\phi_{S}^{\dagger} \phi_{S}\right)+m_{22}^{2}\left(\phi_{D}^{\dagger} \phi_{D}\right)\right]+\frac{\lambda_{1}}{2}\left(\phi_{S}^{\dagger} \phi_{S}\right)^{2} \\
& +\frac{\lambda_{2}}{2}\left(\phi_{D}^{\dagger} \phi_{D}\right)^{2}+\lambda_{3}\left(\phi_{S}^{\dagger} \phi_{S}\right)\left(\phi_{D}^{\dagger} \phi_{D}\right) \\
& +\lambda_{4}\left(\phi_{S}^{\dagger} \phi_{D}\right)\left(\phi_{D}^{\dagger} \phi_{S}\right) \\
& +\frac{\lambda_{5}}{2}\left[\left(\phi_{S}^{\dagger} \phi_{D}\right)^{2}+\left(\phi_{D}^{\dagger} \phi_{S}\right)^{2}\right]
\end{aligned}
$$

In this formulation, all parameters are real (see e.g. [8]).

Depending on the signs and values of the individual parameters in $V\left(\phi_{S}, \phi_{D}\right)$, the minimisation conditions may result in different vacuum configurations where none, one or both vevs of $\phi_{S}$ or $\phi_{D}$ are non-vanishing. Within this work, we focus on the IDM realisation $\left\langle\phi_{S}\right\rangle \neq 0,\left\langle\phi_{D}\right\rangle=0$, for which the decomposition around the vacuum state is given by

$\phi_{S}=\left(\begin{array}{c}\phi^{+} \\ \frac{1}{\sqrt{2}}(v+h+i \xi)\end{array}\right), \phi_{D}=\left(\begin{array}{c}H^{+} \\ \frac{1}{\sqrt{2}}(H+i A)\end{array}\right)$.

Here, $v=246 \mathrm{GeV}$ denotes the SM vacuum expectation value and the scalar field component of $\phi_{S}$ contains the SMlike Higgs boson $h$ with mass

$m_{h}^{2}=\lambda_{1} v^{2}=m_{11}^{2}$,

fixed by the experimentally observed value of $125.1 \mathrm{GeV}$.

In addition to the components known from the Standard Model, the second scalar doublet of the IDM, $\phi_{D}$, contains four dark or inert scalar field components $H, A, H^{ \pm}$with masses given as follows:

$$
\begin{aligned}
m_{H^{ \pm}}^{2} & =\frac{1}{2}\left(\lambda_{3} v^{2}-m_{22}^{2}\right), \\
m_{A}^{2} & =m_{H^{ \pm}}^{2}+\frac{1}{2}\left(\lambda_{4}-\lambda_{5}\right) v^{2}=\frac{1}{2}\left(\bar{\lambda}_{345} v^{2}-m_{22}^{2}\right),
\end{aligned}
$$

$$
m_{H}^{2}=m_{H^{ \pm}}^{2}+\frac{1}{2}\left(\lambda_{4}+\lambda_{5}\right) v^{2}=\frac{1}{2}\left(\lambda_{345} v^{2}-m_{22}^{2}\right),
$$

where we have defined

$\lambda_{345}:=\lambda_{3}+\lambda_{4}+\lambda_{5} ; \bar{\lambda}_{345}:=\lambda_{3}+\lambda_{4}-\lambda_{5}$.

While their interactions with the Standard Model vector bosons can be derived from the gauge kinetic term in the Lagrangian, the absence of any gauge invariant Yukawa-like interaction between $\phi_{D}$ and the Standard Model fermion sector prohibits any tree level interactions between these four dark particles and the SM fermions. Moreover, due to the exact $D$-symmetry the lightest neutral scalar cannot decay and may therefore provide a candidate for dark matter. ${ }^{3}$ Note that, contrarily to generic Two-Higgs-Doublet-Models which denote $H / A$ as the scalar/pseudoscalar components of a doublet, we cannot make such a unique identification here as there is no interaction of $\phi_{D}$ with the Standard Model fermions. In fact, we can swap the roles of $H$ and $A$ by making the replacement $\lambda_{5} \leftrightarrow-\lambda_{5}$, cf. Appendix A.

Within this work, we make the choice $m_{H}<m_{A}, m_{H^{ \pm}}$ and assume $H$ to be the DM candidate. According to Eqs. (5$7)$, this choice implies the relations $\lambda_{5}<0$ and $\lambda_{45}:=\lambda_{4}+$ $\lambda_{5}<0$. The parameters $\lambda_{345}$ and $\bar{\lambda}_{345}$ are related to the triple and quartic coupling between the SM-like Higgs $h$ and the DM candidate $H$ or the scalar $A$, respectively. $\lambda_{3}$ is relevant for the $h$ interaction with the charged scalars $H^{ \pm}$. Lastly, the parameter $\lambda_{2}$ describes the quartic self-couplings of dark particles. A list of all relevant Feynman rules for this model is provided in Appendix A.

Starting from the general scalar potential in Eq. (2), the IDM has 7 degrees of freedom. As $\phi_{S}$ plays the same role as the SM Higgs doublet for electroweak symmetry breaking, the two parameters $m_{h}$ and $v$ are fixed by the Higgs mass measurement and electroweak precision data, respectively. We are therefore left with 5 degrees of freedom which we choose to be the physical parameters

$\left(m_{H}, m_{A}, m_{H^{ \pm}}, \lambda_{2}, \lambda_{345}\right)$.

From these the corresponding dependent values of the other theory parameters can be derived by applying the relations given above.

\section{Constraints}

As has been widely discussed in the literature, the IDM is subject to numerous constraints which can be derived from both theoretical grounds as well as experimental results. We briefly remind the reader of these constraints here and refer to

\footnotetext{
${ }^{3}$ Charged DM has been strongly limited by astrophysical analyses [46].
} 
the literature $[2,4-6,15,21-23,28,30-33,45,47-67]$ for further details.

The constraints we use in this work have been extensively discussed in Refs. [22,23,45] and we refer the reader to these references for more detailed explanations. Here, we only summarise all relevant constraints and point to updates on experimental limits whenever applicable. The calculation of the IDM spectrum and tests of several of the below bounds have been obtained using $2 \mathrm{HDMC}$ [68].

\subsection{Theoretical constraints}

We apply the following theoretical constraints:

- The vacuum of the model needs to be bounded from below. ${ }^{4}$ These lead to the conditions

$$
\lambda_{1}>0, \lambda_{2}>0, \lambda_{3}+\sqrt{\lambda_{1} \lambda_{2}}>0, \lambda_{345}+\sqrt{\lambda_{1} \lambda_{2}}>0
$$

- All couplings must allow for a perturbative discussion which is why we restrict all couplings to be smaller than $4 \pi$.

- All $2 \rightarrow 2$ scalar scattering processes must not violate perturbative unitarity and we apply standard bounds as implemented in $2 \mathrm{HDMC}$.

- In generic Two Higgs Doublet Models, several vacua can coexist. The tree level condition to be in the inert vacuum has been calculated in $[8,55,56]$

$$
\frac{m_{11}^{2}}{\sqrt{\lambda_{1}}} \geq \frac{m_{22}^{2}}{\sqrt{\lambda_{2}}}
$$

Here, $m_{11}$ and $m_{22}$ can directly be derived from Eqs. (4)(7) and the above condition translates to

$$
\lambda_{345} \leq \frac{\sqrt{\lambda_{2}} m_{h} v+2 m_{H}^{2}}{v^{2}}
$$

The above constraint links the value of $\lambda_{345}$ and the dark scalar mass to the coupling $\lambda_{2}$ which describes selfcouplings in the scalar sector and has no influence on collider phenomenology (see e.g. the discussion in [45]). Requiring the Higgs self-coupling vertices to acquire maximally allowed values of $4 \pi$ leads e.g. to $\lambda_{2} \lesssim 4$ [22]. This bound, in the parameter region with relatively light dark scalars with masses $m_{H} \lesssim 100 \mathrm{GeV}$, would result in $\lambda_{345} \leq \mathcal{O}(1)$. Note, however, that this bound is not completely mandatory. Several minima may coexist (see e.g. $[69,70]$ ) and the inert one may only be a

\footnotetext{
4 The conditions are applied at tree level; see e.g. Refs. $[19,35]$ for a discussion of changes using higher-order predictions.
}

local one as long as the transition time to the global noninert minimum is sufficiently large. Moreover, the above condition may be significantly altered at next-to-leading order, see e.g. Refs. [60,71]. The next-to-leading order effects are however quite involved and can not easily be generalized, but need to be recalculated on a case-by-case basis ${ }^{5}$. In this work, we focus on current constraints from LHC searches that are independent of $\lambda_{2}$; we therefore also consider values of $\lambda_{345} \gtrsim 1$. In case of a discovery, a detailed analysis would be needed in order to correctly evaluate the above condition beyond leading order, see e.g. related studies in Refs. [71,72].

\subsection{Experimental constraints}

In addition to the theoretical bounds listed above, several experimental observations put tight constraints on the parameter space of the IDM:

- We fix the mass of the SM-like Higgs boson $h$ to

$$
m_{h}=125.1 \mathrm{GeV}
$$

in agreement with the results from the LHC experiments [73]. Note that this has already been accounted for when we chose $\left(m_{H}, m_{A}, m_{H^{ \pm}}, \lambda_{2}, \lambda_{345}\right)$ as the five degrees of freedom of the IDM.

- We furthermore require the total width of the $125 \mathrm{GeV}$ Higgs to obey Ref. [74]

$$
\Gamma_{\text {tot }} \leq 9 \mathrm{MeV}
$$

which is applicable in those regions of parameter space which predict additional decays of the SM-like Higgs boson.

- Furthermore, we take into account strong bounds from the measured total widths of the electroweak SM gauge bosons, cf. e.g. Ref. [75], by forbidding potentially dangerous kinematic mass configurations via the following hard constraints:

$$
\begin{aligned}
m_{A, H}+m_{H^{ \pm}} & \geq m_{W}, \\
m_{A}+m_{H} & \geq m_{Z}, \\
2 m_{H^{ \pm}} & \geq m_{Z} .
\end{aligned}
$$

- We furthermore require a $2 \sigma$, i.e. $95 \%$ C.L., agreement with electroweak precision observables, parameterized through the electroweak oblique parameters $S, T$ and $U$ [76-79].

\footnotetext{
5 We thank P. Ferreira and B. Swiezewska for useful discussions regarding this point.
} 
- In order to evade bounds from long-lived charged particle searches, we conservatively set an upper limit on the charged scalar lifetime of $\tau \leq 10^{-12} s$, to guarantee decay before the innermost detector layer. This translates to a lower bound on the total decay width of the charged scalar $H^{ \pm}$of $\Gamma_{\text {tot }} \geq 6.58 \times 10^{-13} \mathrm{GeV}$. Mass dependent bounds on the charged scalar lifetime have been studied in detail in Ref. [67].

- A bound on the lower mass of $m_{H^{ \pm}}$has been derived in Ref. [80]. Although a more dedicated analysis of this bound within the current models' framework would be required, we take $m_{H^{ \pm}} \geq 70 \mathrm{GeV}$ as a conservative lower limit.

- We also require agreement with the null-searches from the LEP, Tevatron, and LHC experiments using HiggsBounds - 5.2.0beta [81-84], including all experimental bounds up to Moriond 2017. ${ }^{6}$

- We update the limits on the invisible decay of $m_{h}$ and take the results presented in Ref. [86] which require $\mathrm{BR}_{h \rightarrow \text { inv }} \leq 0.24$.

- Furthermore, we apply new limits on the branching ratio $h \rightarrow \gamma \gamma$ taken from [87] and require $\mu=1.14_{-0.18}^{+0.19}$. Since within the IDM the production cross sections of the SM-like Higgs are unaffected, we use the bound on $\mu$ in combination with the Standard Model value [39] of $\mathrm{BR}(h \rightarrow \gamma \gamma)=2.270 \times 10^{-3}$ and require

$$
\mathrm{BR}(h \rightarrow \gamma \gamma) \in[1.77 ; 3.45] \times 10^{-3}
$$

at the two-sigma level.

- In addition, we require agreement within $2 \sigma$ for the 125 $\mathrm{GeV}$ Higgs signal strength measurements. For this, we make use of the publicly available tool HiggsSignals-2.2.1beta [88], and require $\Delta \chi^{2} \leq$ 11.3139 , corresponding to the $95 \%$ confidence level of a 5-dimensional fit. $^{7}$

- We also include limits on the model's parameter space that have been obtained in previous reinterpretations of collider dark matter searches, predominantly within supersymmetric scenarios. Major limits stem from the reinterpretation of a LEP analysis [40] within the IDM framework [52]. This particularly rules out all regions where

$$
\begin{aligned}
m_{A} & \leq 100 \mathrm{GeV}, \\
m_{H} & \leq 80 \mathrm{GeV}, \\
\Delta m(A, H) & \geq 8 \mathrm{GeV}
\end{aligned}
$$

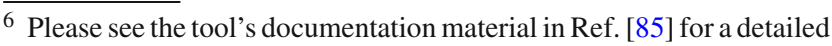
discussion of the included limits.

7 We used a combination of Run I combination, Run II, and simplified template cross sections within Higgs Signals.
}

are simultaneously fulfilled.

- After taking into account all the above limits we are outside of the region excluded due to the recent reinterpretation of the SUSY analysis from LHC Run I [20].

- We apply dark matter relic density limits obtained by the Planck experiment [89]:

$\Omega_{c} h^{2}=0.1200 \pm 0.0012$

In this work, we do not require the dark matter candidate of the IDM to provide the full relic density, but use it as an upper limit. ${ }^{8}$ Being conservative, we require

$\Omega_{c} h^{2} \leq 0.1224$

which corresponds to not overclosing the universe at $95 \%$ confidence level. In addition to this bound, we specifically identify those regions which reproduce the observed DM density within the $2 \sigma$ interval around the above best fit value. The dark matter relic density has been calculated using MicrOmegas version 4.3.5 [91].

- Regarding direct detection dark matter constraints, we compare to the most recent results of XENON1T [92]. ${ }^{9}$

As before, we consider the possibility of a multi- component dark matter scenario in which the IDM only makes up for a fraction of the total dark matter relic density. In this case, the upper limit from direct detection depends on the actual DM relic density for the specific point in parameter space; therefore, we have to introduce a rescaling factor, leading to the (relic density dependent) limit

$\sigma\left(m_{H},\{\ldots\}\right) \leq \sigma^{\mathrm{XENON} 1 \mathrm{~T}}\left(m_{H}\right) \times \frac{\Omega^{\text {Planck }}}{\Omega\left(m_{H},\{\ldots\}\right)}$,

where $m_{H}$ now denotes the dependence on the mass of our dark matter candidate $H$ and $\{\ldots\}$ is short for all other parameters specifying the respective IDM parameter point. ${ }^{10}$ Direct detection cross sections are again obtained using MicrOmegas.

The scan setup has been described in great detail in Ref. [22]. To determine allowed regions in parameter space,

\footnotetext{
8 In such a scenario, additional dark matter candidates would be needed in order to account for the missing relic density; cf. e.g. Ref. [90] for a dedicated discussion of such scenarios within a supersymmetric setup.

9 We here use the data available from Ref. [93] in a digitalized format. In our code, we use an approximation function which reproduces these constraints on the per-cent level.

${ }^{10}$ See also Refs. [90,94-97].
} 
we follow the procedure discussed therein, including the experimental updates listed above.

\section{LHC analysis of VBF and monojets}

In this work, we choose to constrain ourselves to cases for dark matter candidate masses $m_{H} \leq 100 \mathrm{GeV}$. Due to the relatively high production cross section in such cases, these will be the regions which are most sensitive to collider searches (see e.g. [30,32,33,98] for recent work on low mass scenario studies at the LHC).

We here concentrate on the $13 \mathrm{TeV}$ CMS search for an invisibly decaying Higgs [41] produced through vector boson fusion (VBF) and a $13 \mathrm{TeV}$ ATLAS search [42] for dark matter candidates in the monojet channel. These respectively lead to the collider signatures

$$
\begin{aligned}
& p p \rightarrow j j+\mathbb{E}_{T} \quad(\mathrm{VBF}), \\
& p p \rightarrow j+\mathbb{E}_{T} \text { (Monojet). }
\end{aligned}
$$

In this study, we mainly focus on the VBF channel which, as we show later, provides the strongest sensitivity. We however also determine bounds on the IDM using a monojet reinterpretation for comparison. A dedicated exploration of this channel including sensitivity prospects of the high luminosity LHC can be found in Ref. [32].

\subsection{Features of the VBF channel}

The two jets in the VBF channel typically have a large separation in pseudorapidity. The corresponding cuts used in the above analysis are listed in Table 1. These form a "Cutand-Count analysis" and a "Shape analysis". The former is designed for a large signal-to-background ratio and requires a large value for the invariant mass $m_{j j}$ of the jet pair, whilst the latter defines several signal regions binned in $m_{j j}$ and used collectively in a fit. Using these signatures, the CMS collaboration finds an upper limit on the invisible Higgs branching ratio of $\mathrm{BR}_{h \rightarrow \text { max }}^{\max }=0.53$ using the cut-and-count analysis and 0.28 for the shape analysis which are both weaker than the upper limit $\mathrm{BR}_{h \rightarrow \text { inv }}^{\max }=0.24$ used as a hard cut in our scan (see Sect. 3.2). This constraint is only applicable to parameter points in the IDM for which $m_{H}<m_{h} / 2$. However, points with heavier scalars would also predict additional signal events in the above analysis due to processes with off-shell $h$ production ( $p p \rightarrow h^{*} j j \rightarrow H H j j$ ) and contributions from decay chains with hadronically decaying final state particles (e.g. $p p \rightarrow H^{ \pm} H \rightarrow j j H H$ ). We recast the above mentioned VBF analysis in the context of these processes to potentially extract additional constraints applicable to regions with larger values of $m_{H}$.
Table 1 Summary of the main kinematic requirements in the signal regions in Ref. [41]

\begin{tabular}{lcc}
\hline \hline Requirement & Cut-and-Count & Shape \\
\hline Leading Jet $p_{\mathrm{T}}$ & $>80 \mathrm{GeV}$ \\
Second Jet $p_{\mathrm{T}}$ & $>40 \mathrm{GeV}$ \\
$E_{\mathrm{T}}^{\text {miss }}$ & $>250 \mathrm{GeV}$ \\
$\left|\Delta \phi_{j, E_{\mathrm{T}}^{\text {miss }}}\right|$ & $>0.5$ \\
$\left|\Delta \phi_{j j}\right|$ & $<1.5$ \\
$\eta_{1} \cdot \eta_{2}$ & $<0$ & \\
$\left|\Delta \eta_{j j}\right|$ & $>4.0$ & $>1.0$ \\
$m_{j j}$ & $>1.3 \mathrm{TeV}$ & $>200 \mathrm{GeV}$ (binned) \\
\hline \hline
\end{tabular}

\subsection{Simulation and validation of the VBF channel}

In this work, we concentrate on the above VBF search which has been implemented within the CheckMATE $[43,44]$ framework. CheckMATE uses simulated event files for any BSM model, applies detector efficiencies and follows the event selection procedure of the implemented BSM searches from ATLAS and CMS to determine if any resulting signal prediction would violate the corresponding experimental bound. ${ }^{11}$ Validation has been performed by reproducing the quoted numbers expected from the Standard Model Higgs boson with $100 \%$ invisible branching ratio. Following the procedure described in the experimental publication, we use the POWHEG-Box [100-103] for simulating Monte-Carlo events at next-to-leading order in QCD and subsequently interface it to Pythia 6.4.21 [104] to account for parton showering and hadronization of the final state. We perform the simulation separately for vector-boson-fusion (vbf) and gluon-initiated final states (ggf) which may also pass the aforementioned cuts.

As we are bound to leading order Monte Carlo tools for the simulation of the IDM, we additionally generate treelevel parton events with MG5_aMC@NLO [105] showered with Pythia 8.219 [106] - the same tools which we use for our subsequent IDM analysis - to quantify the effect of an LO-only simulation. Both event samples are processed with CheckMATE and the resulting signal predictions are shown in Table 2.

As can be seen, our setup reproduces the experimentally quoted results sufficiently well within the experimentally quoted error margin when using simulated events generated with an NLO-QCD Monte Carlo event generator. A leading-order Monte Carlo analysis, in comparison, signif-

\footnotetext{
${ }^{11}$ For more information about how CheckMATE works we refer to the corresponding manuals in Refs. [43,44]. We implemented the above mentioned VBF search using the AnalysisManager tool described in Ref. [99].
} 
Table 2 Observed and expected number of events for all regions listed in Table 1. SM predictions are determined for an entirely invisibly decaying Standard Model Higgs boson with $m_{h}=125 \mathrm{GeV}$ produced both in Vector Boson Fusion and Gluon Fusion. CMS numbers are taken from Ref. [41] and compared to our numbers determined with our analysis implementation in CheckMATE, using both the LO-QCD generator MG5_aMC@NLO and the NLO-QCD Monte Carlo simulation Powheg-Box. Uncertainties quoted for CMS include both statistical and systematical uncertainties

\begin{tabular}{|c|c|c|c|c|c|c|}
\hline \multirow[t]{3}{*}{ Region } & \multirow[t]{3}{*}{ Data } & \multirow{3}{*}{ Background } & \multicolumn{4}{|c|}{ SM prediction with $\mathrm{BR}(h \rightarrow$ invisible $)=100 \%$} \\
\hline & & & \multirow[t]{2}{*}{ CMS } & \multicolumn{3}{|c|}{ Our Simulation } \\
\hline & & & & Powheg-Box & MG5_aMC@NLO & Ratio \\
\hline CutandCount & 2053 & $1779 \pm 96$ & $851 \pm 148$ & 758 & 468 & 1.6 \\
\hline$m_{j j} \in[200,400] \mathrm{GeV}$ & 16177 & $14878 \pm 566$ & $591 \pm 285$ & 708 & 390 & 1.8 \\
\hline$m_{j j} \in[400,600] \mathrm{GeV}$ & 10008 & $9401 \pm 387$ & $571 \pm 232$ & 664 & 374 & 1.8 \\
\hline$m_{j j} \in[600,900] \mathrm{GeV}$ & 7277 & $6658 \pm 271$ & $566 \pm 172$ & 737 & 433 & 1.7 \\
\hline$m_{j j} \in[900,1200] \mathrm{GeV}$ & 3138 & $2994 \pm 144$ & $472 \pm 131$ & 483 & 293 & 1.7 \\
\hline$m_{j j} \in[1200,1500] \mathrm{GeV}$ & 1439 & $1283 \pm 69$ & $307 \pm 64$ & 314 & 202 & 1.7 \\
\hline$m_{j j} \in[1500,2000] \mathrm{GeV}$ & 911 & $834 \pm 51$ & $344 \pm 83$ & 319 & 203 & 1.6 \\
\hline$m_{j j} \in[2000,2750] \mathrm{GeV}$ & 408 & $358 \pm 29$ & $228 \pm 40$ & 218 & 126 & 1.8 \\
\hline$m_{j j} \in[2750,3500] \mathrm{GeV}$ & 87 & $73.8 \pm 9.4$ & $90.3 \pm 18.8$ & 80.1 & 48.8 & 1.7 \\
\hline$m_{j j}>3500 \mathrm{GeV}$ & 29 & $30.3 \pm 7.4$ & $37.4 \pm 9.1$ & 38.2 & 19.9 & 1.9 \\
\hline
\end{tabular}

icantly underestimates the signal prediction with a nearly constant ratio of $\approx 1.7$ across all signal regions.

The numbers in Table 2 can be used to derive upper limits on the invisible branching ratio of the Standard Model Higgs. For this purpose we employ a profile likelihood ratio test paired with the CLs prescription. For the shape analysis, we make use of the full background covariance matrix provided in Ref. [41]. As no such detailed information is provided for the signal, we conservatively assume that it is fully correlated across all bins. Our resulting distribution for the test statistics is shown in Fig. 1. We are able to reproduce this distribution sufficiently well by either using our results determined with POWHEG or by rescaling the results of our leading-order simulation with a constant $K$-factor of 1.7.

From our validation and the comparison of the above results using LO and NLO simulation, we conclude that a leading order simulation of the IDM signal is expected to systematically underestimate the correct number. However, a full next-to-leading order simulation of the off-shell VBF channel within the IDM is beyond the scope of this work. We determine results for the IDM at leading order. To be more precise, we simulate

$p p \rightarrow H H j j$

event samples for the IDM within MG5_aMC@NLO and Pythia 8.219 by making use of the UFO model description of Ref. [19]. We here do not specify intermediate states, i.e. on parton level all processes leading to the final state given in Eq. (23) have been taken into account. Besides VBF production of a SM-like scalar with invisible decays, this also includes e.g. $H A$ or $H^{ \pm} H$ pair-production with the subsequent decay $A \rightarrow H j j, H^{ \pm} \rightarrow H j j$ or vector boson scattering processes with dark scalars in the $t$-channel. Rel-

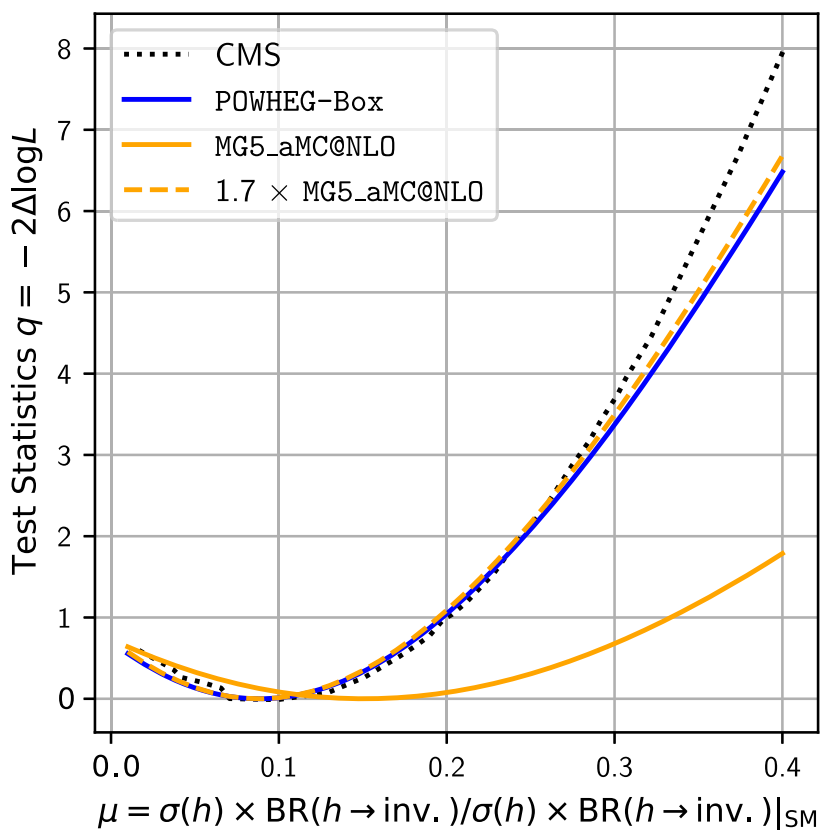

Fig. 1 Comparison of the log-likelihood-ratio, using the numbers in Table 2

ative contributions of the latter to the total cross section become sizeable as $\left|\lambda_{345}\right| \rightarrow 0$. We include an invariant mass cut $m_{j j} \geq 130 \mathrm{GeV}$ and a pseudorapidity difference cut $\Delta \eta_{j j} \geq 0.5$ with $\eta_{1} \eta_{2}<0$ in our parton event generation. Note that these are weaker than the signal region cuts in Table 1.

From our above findings, we expect our resulting bounds to be conservative. However, motivated from the results in Table 2 we also discuss the limits we obtain if our signal prediction is upscaled with the global $K$-factor of 1.7 motivated 
before to illustrate the potential impact of next-to-leadingorder QCD effects.

\subsection{Features and setup of the monojet analysis}

Nearly any particle model with a dark matter candidate $H$ predicts the standard monojet signature $p p \rightarrow H H j$ for the LHC where the jet may originate from initial state radiation or, in some specific models other than ours, from the hard vertex. It is therefore to be expected that this channel is sensitive to the IDM in which $H$ plays the role of the dark matter candidate. A detailed analysis of this channel can be found in Ref. [32]. However, as has for example been shown in Ref. [107] in the context of a different model with similar topology, the vector boson fusion channel is expected to be significantly more sensitive than the monojet search. We reproduce this finding later.

The analysis of the monojet channel is performed within CheckMATE, similarly to the analysis above. As this analysis had already been implemented in the public code, we do not provide a separate validation here. ${ }^{12}$ We simulate the partonic process $q \bar{q}, g g \rightarrow H H j$ with MG5_aMC@NLO and apply a $p_{T}$ cut of $200 \mathrm{GeV}$ on the leading jet, in accordance with the signal region requirement $p_{T}^{j} \geq 250 \mathrm{GeV}$ of this analysis.

\section{Parameter space constraints}

In this section, we present the constraints resulting from the our recast of the searches for an invisibly decaying Higgs in both the vector boson fusion and the monojet channel. We initially consider parameter points which have passed all bounds presented in Sect. 3, apart from the constraints imposed by dark matter bounds, i.e. dark matter relic density as well as direct detection, cf. Eqs. (19) and (20). This approach allows for an investigation of the complementary between astrophysical and collider searches for this model.

\section{Collider constraints}

We now demonstrate the effect of including the searches in Refs. [41], [42] as introduced in the previous section. Our results are shown in Fig. 2 (top) where we only consider points which pass all prior constraints discussed in Sect. 3. ${ }^{13}$ The general influence of these constraints has been discussed

\footnotetext{
12 Validation material for this analysis can be found on the official CheckMATE website, https://checkmate.hepforge.org/AnalysesList/ ATLAS_13TeV.html.

13 We note that the density of points has no theoretical meaning but is just a reflection of a bias in the generation of theoretical parameter tuples.
}
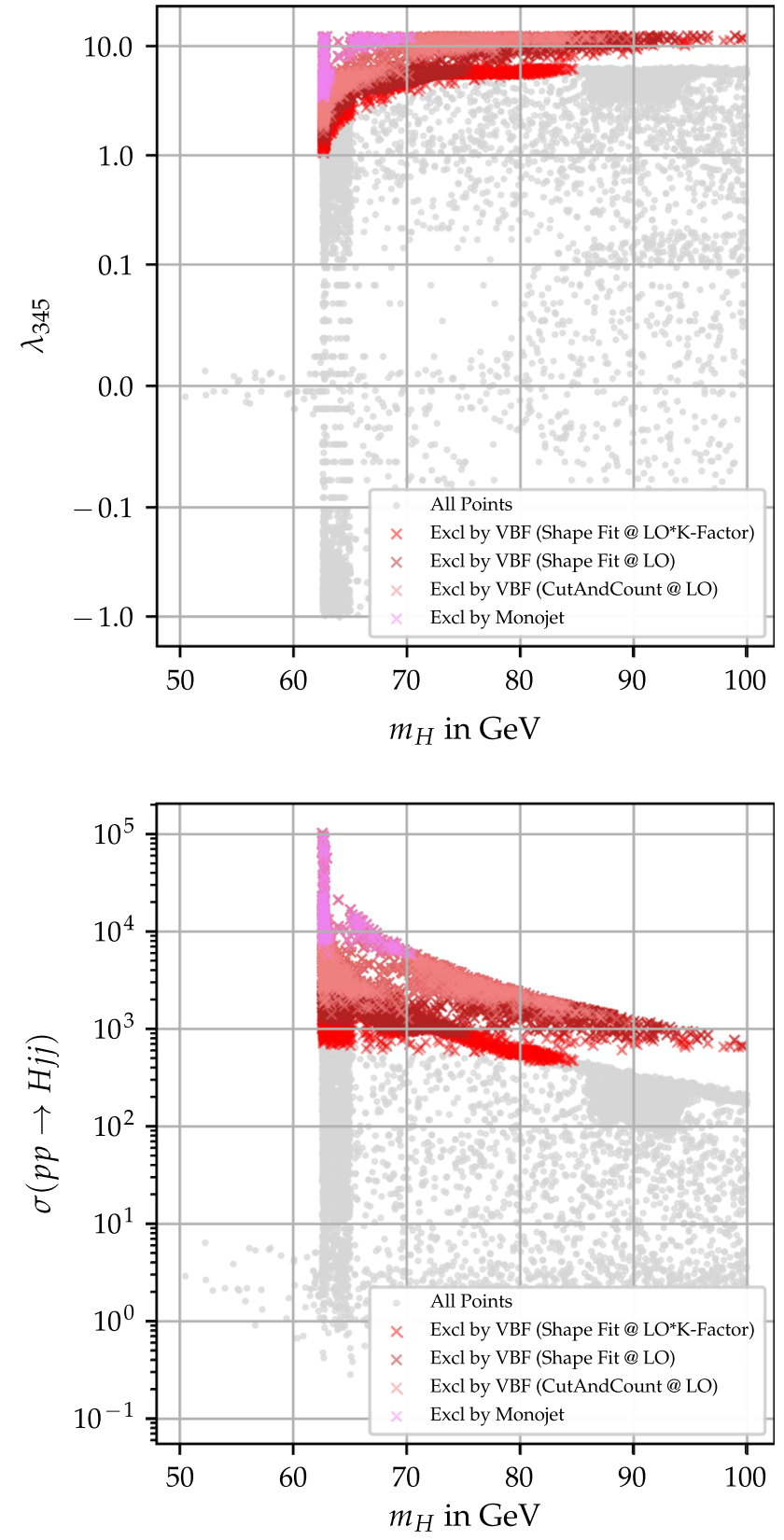

Fig. 2 Allowed and excluded points after consideration of VBF and monojet analysis but without dark matter relic density and direct detection constraints. VBF results are shown using different categories, see in-text discussion. Top: Results in $\left(m_{H}, \lambda_{345}\right)$ parameter plane. Bottom: Results in $m_{H}-\sigma$ plane where $\sigma$ is the LHC VBF production cross section at $13 \mathrm{TeV}$ including the partonic cuts given in Sect. 4.2

in detail in Refs. [22,45] and will not be repeated here. For values $m_{H} \leq m_{h} / 2$, it is especially the branching ratio limit on $h \rightarrow$ invisible which leads to the tight constraint $\left|\lambda_{345}\right| \lesssim$ 0.03 . For larger $m_{H}$ values, however, $\lambda_{345}$ can reach values up to the perturbativity limit $4 \pi$ which has been imposed as a hard upper cut in the scan setup. Note that we have explicitly verified that the small stripe for $m_{H}>80 \mathrm{GeV}$, $\lambda_{345}>6$ contains no viable parameter points as it is excluded 
by combining perturbativity requirements with limits on the electroweak oblique parameters and $R_{\gamma \gamma}$, see e.g. discussion in Ref. [22].

We separately indicate which points are respectively excluded by the monojet and by the VBF search. For the latter, we explicitly distinguish the following exclusion categories.

- In CutAndCount@LO, we only determine the number of signal events in the cut-and-count signal region of Ref. [41] and use a single-bin likelihood ratio test to determine whether it is compatible with the numbers of observed and expected Standard Model events, see Table 2.

- In Shape FitaLO we determine the number of signal events in all $m_{j j}$ binned signal regions of Table 2 and use a joint likelihood, including the background correlation matrix provided in Ref. [41], to determine the overall $p$-value.

- Whilst for the above two approaches we use the signal numbers as determined with the Monte Carlo generator MG5_aMC@NLO at leading order, for Shape Fit@LO*K- Factor we multiply all numbers with the constant $K$-factor of 1.7 , c.f. discussion in Sect. 4.2.

According to our SM validation, we expect $\mathrm{LO}$ results to significantly underestimate the number of signal events and therefore lead to conservative bounds. Showing the results including the $K$-factor determined from our SM validation renders an estimate of the impact of higher-order QCD contributions.

In general, we observe that a significant fraction of points can be constrained by the two collider searches considered in this work. As foreseen in Sect. 4.3, the monojet channel shows a significantly reduced sensitivity as compared to the VBF search. ${ }^{14}$ Though both channels suffer largely from SM QCD background sources, the VBF channel can make more precise predictions on the expected kinematics of the jets in the final state. This ultimatively allows for a higher signalto-background ratio in the signal bins and thus results in a better sensitivity for many models in which both channels are present simultaneously.

Whilst monojet studies alone are sensitive to values of $\lambda_{345}$ down to 2.5 and $m_{H}$ masses in the range $\left[m_{h} / 2-70\right] \mathrm{GeV}$, we observe VBF reinterpretations to constrain $\lambda_{345}$ down to 1 and extend the sensitivity range on $m_{H}$ values up to the maximum of $100 \mathrm{GeV}$ we consider. No parameter point with $m_{H}<m_{h} / 2$ can be constrained as the small values of $\lambda_{345}$

\footnotetext{
14 Our results appear to be compatible with former monojet sensitivity studies shown in Ref. [32] which show no sensitivity for a benchmark point with $\lambda_{345}=1.7$ using an older version of the monojet search with only $10 \%$ of the integrated luminosity that our analysis uses.
}

predict a far too small cross section. This can also be seen on the bottom of Fig. 2 where we show our bounds in terms of the VBF cross section, including the partonic cuts described in Sect. 4.2. In fact, this region is largely constrained by the cut on $\mathrm{BR}_{h \rightarrow \text { inv }} \leq 0.24$ which we discuss in Sect. 3.2. As the VBF channel consists of one sub-measurement of this observable, it is evident that it cannot provide additional, stronger bounds than the one on the invisible branching ratio which has been used to generate our parameter samples.

Note that, though the bound is clearly very much dependent on the size of the cross section, we observe on the bottom of Fig. 2 that it is not flat in the $m_{H}-\sigma$-plane. This can be explained by differences in the signal efficiency from additional, small IDM contributions like $p p \rightarrow H^{ \pm} H, H^{ \pm} \rightarrow$ jj $H$ which, in addition to $m_{H}$, also depend on the masses of the other inert scalars. The fact that the bound is not only dependent on the total cross section shows the importance of dedicated Monte Carlo recast analyses including off-shell effects.

\section{Dark matter constraints}

We now impose the dark matter constraints specified by Eqs. (19) and (20) on the parameter space. As has been noted in Refs. [23,45], it is especially direct detection constraints which have improved by an order of magnitude with respect to the previous study in Ref. [22] which used the 2013 LUX results, c.f. Ref. [108]. The parameter space is severely constrained, as is demonstrated in Fig. 3 where we now discuss the dark matter bounds on our parameter space without applying the VBF/Monojet limits. Fig. 3, top, shows the results in the $m_{H}-\lambda_{345}$ plane and Fig. 3, bottom, displays the relic density abundance $\Omega_{c} h^{2}$ in dependence on $m_{H}$. The second figure also labels the dominant annihilation channel for each tested parameter point as determined via MicrOmegas. ${ }^{15}$ We also indicate the point in our sample whose predicted value of $\Omega_{c} h^{2}=0.1141$ is closest to the Planck value in Eq. (18). This point yields $95 \%$ of the required cold dark matter relic density. Especially for masses $m_{H} \geq 63 \mathrm{GeV}$, we find that $\left|\lambda_{345}\right|$ needs to be small, $\lesssim 0.14$ for $m_{H} \approx 100$ $\mathrm{GeV}$ and even tighter bounds for lighter $m_{H}$. However, there also exists a small mass window, $m_{H} \in\left[m_{h} / 2 ; 63 \mathrm{GeV}\right]$ which allows for values of $\lambda_{345}$ up to our theoretical limit of $4 \pi$. As can be seen in the bottom of Fig. 3 , this region predicts

\footnotetext{
15 For relatively small mass differences between the two dark neutral scalars $A$ and $H$, typically of a few $\mathrm{GeV}$, the co-annihilation channel A $H \rightarrow d \bar{d}$ becomes dominant. As this requires a relatively fine-tuned scenario, our scan only tested 2 such points. See also the discussion in $[22,98]$.
} 

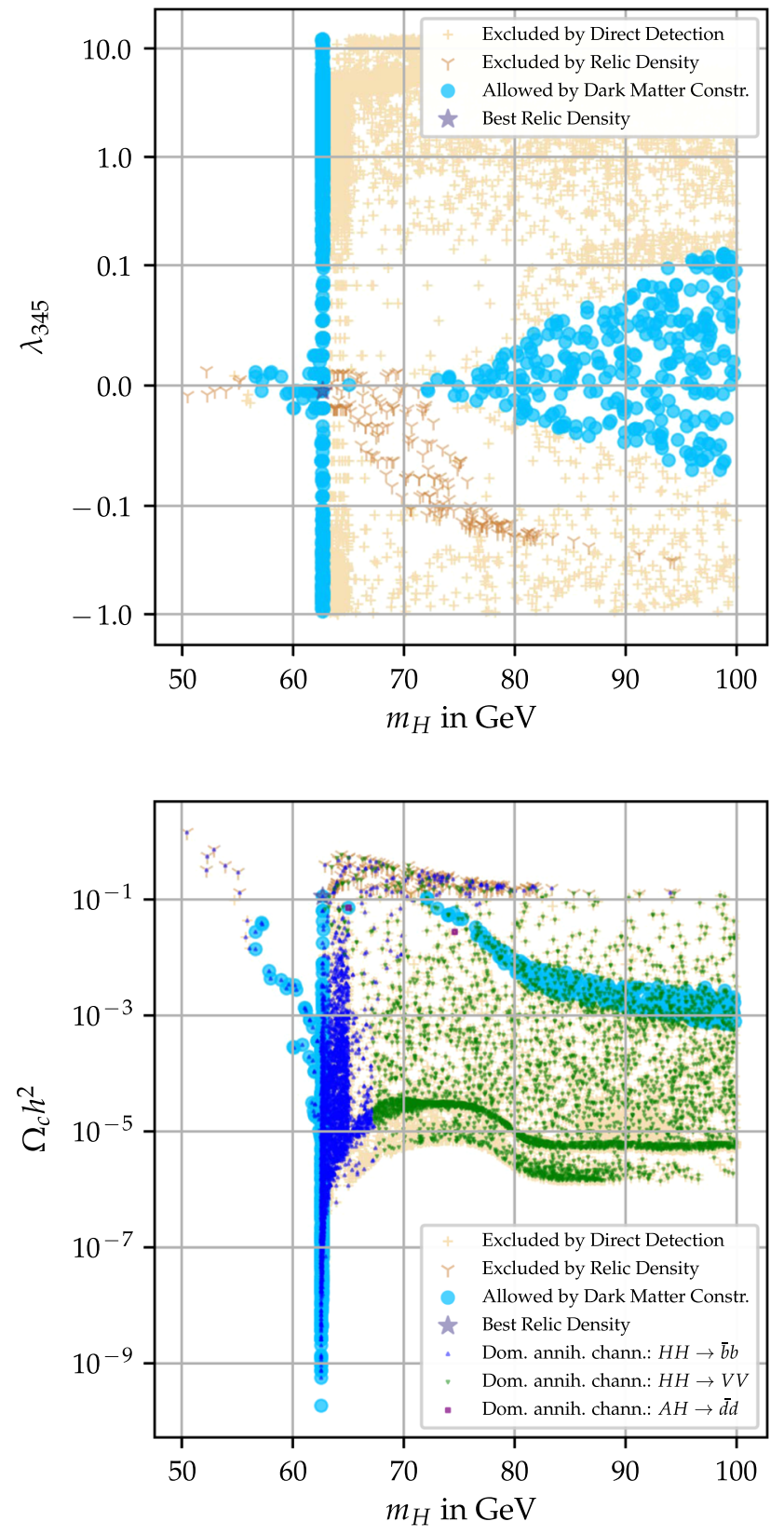

Fig. 3 Parameter space after including dark matter relic density and direct detection constraints. Top: allowed and forbidden regions in the $\left(m_{H}, \lambda_{345}\right)$ plane. Bottom: Constraints in the $\left(m_{H}, \Omega_{c} h^{2}\right)$ plane. On the bottom plot, we also show the dominant annihilation cross section for each parameter point. The "Best Relic Density" point yields $\Omega_{c} h^{2}=$ 0.1141 which is the closest to the nominal Planck value, c.f. Eq. (18), out of all tested points

particularly small values of $\Omega$ and therefore avoids both relic density and direct detection constraints, see Eq. (20). When the intermediate SM-like Higgs boson $h$ is on shell, the annihilation cross section $H H \rightarrow b \bar{b}$ is enhanced and results in a considerably smaller dark matter relic density (see also discussion in $[22,98])$.
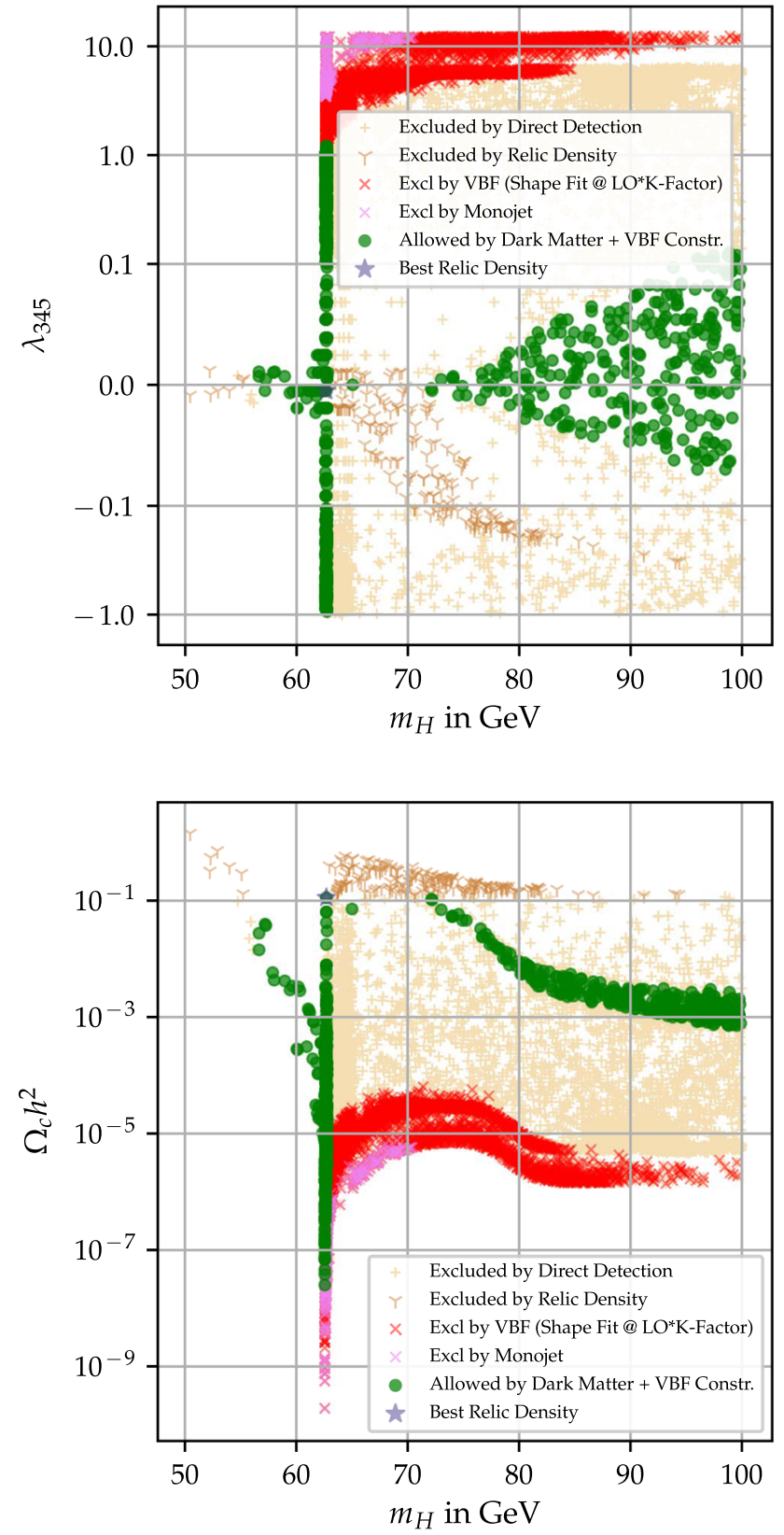

Fig. 4 Parameter space after including all constraints (see also explanations below Fig. 3)

\section{Combination}

Finally, in Fig. 4, we show the allowed and excluded parameters $m_{H}$ and $\lambda_{345}$, as well as $\Omega_{c} h^{2}$, after all the above constraints are taken into account. As can be seen, collider results start to close the annihilation window, $m_{H} \approx m_{h} / 2$, which could bypass direct detection constraints by significantly reducing the predicted relic density $\Omega_{c} h^{2}$. Here, only collider searches can put bounds on values of $\lambda_{345}$ above 1 . However, this only constrains points with very small $\Omega_{c} h^{2}$; therefore still a large number of points in this kinematic 
window remain allowed, including our "Best Relic Density" point discussed above. Moreover, for values of $m_{H}$ significantly larger than $m_{h} / 2$, collider limits may yield important bounds. However in the IDM we find that these are always already excluded by direct detection limits.

It must be noted, though, that if the lightest IDM scalar $H$ couples to an extended dark sector and in fact decays to the actual, lighter dark matter candidate, relic density and direct detection constraints can change significantly while the above collider bounds are typically unaffected if $H$ has further invisible decays (see e.g. Ref. [109] in the context of the so-called "radiative seesaw model" which extends the IDM with an additional Majorana neutrino dark matter candidate). Therefore, even though in the pure IDM collider limits seem to hardly provide additional sensitivity compared to direct detection limits, they still constitute an important analysis channel complementary to dark matter findings.

\section{Null results from other recast channels}

The above VBF and monojet analyses focus on the dark matter candidate $H$ and thus are largely independent of the masses $m_{A}, m_{H^{ \pm}}$of the other two scalar particles and their decay rates. However, within our scan we only considered dark masses $\leq 500 \mathrm{GeV}$. Thus the question may arise if any of the many other BSM searches performed by ATLAS and CMS could result in additional, stronger constraints than the one considered.

In Fig. 5 we display the masses $m_{H^{ \pm}}, m_{A}$ for all points that are allowed by our previous scan. Similar to findings in Refs. [22,45], we observe a relatively strong mass degeneracy of these two heavier dark scalars. We also show the corresponding mass differences $m_{A}-\left(m_{H}+m_{Z}\right)$ and $m_{H^{ \pm}}-\left(m_{H}+m_{W^{ \pm}}\right)$of the allowed points. This quantity can be used to roughly estimate the kinematics of the expected decays for the heavier scalars $A$ and $H^{ \pm}$. For mass differences larger/smaller than 0 , we expect on/off-shell decays into gauge bosons, e.g. $A \rightarrow H Z^{(*)}$, with $100 \%$ branching ratio due to the absence of any other lighter $D$-odd particles. We focus on the leptonic decay modes of the gauge bosons as within the analyses we consider, hadronic modes are typically harder to distinguish from QCD background.

The larger the mass difference, the more energy is expected to be passed on to the daughter particles. A highmomentum $H$ in the final state is expected to produce missing transverse momentum (MET) in the event, a key observable for BSM signals. However, as can be seen in Fig. 5, viable IDM points only predict large mass differences for parameter points if also the absolute masses of $m_{A}$ and $m_{H^{ \pm}}$ are increased. Points with a large mass splitting and good final state efficiency therefore in turn suffer from respectively smaller expected LHC production cross sections. Therefore,
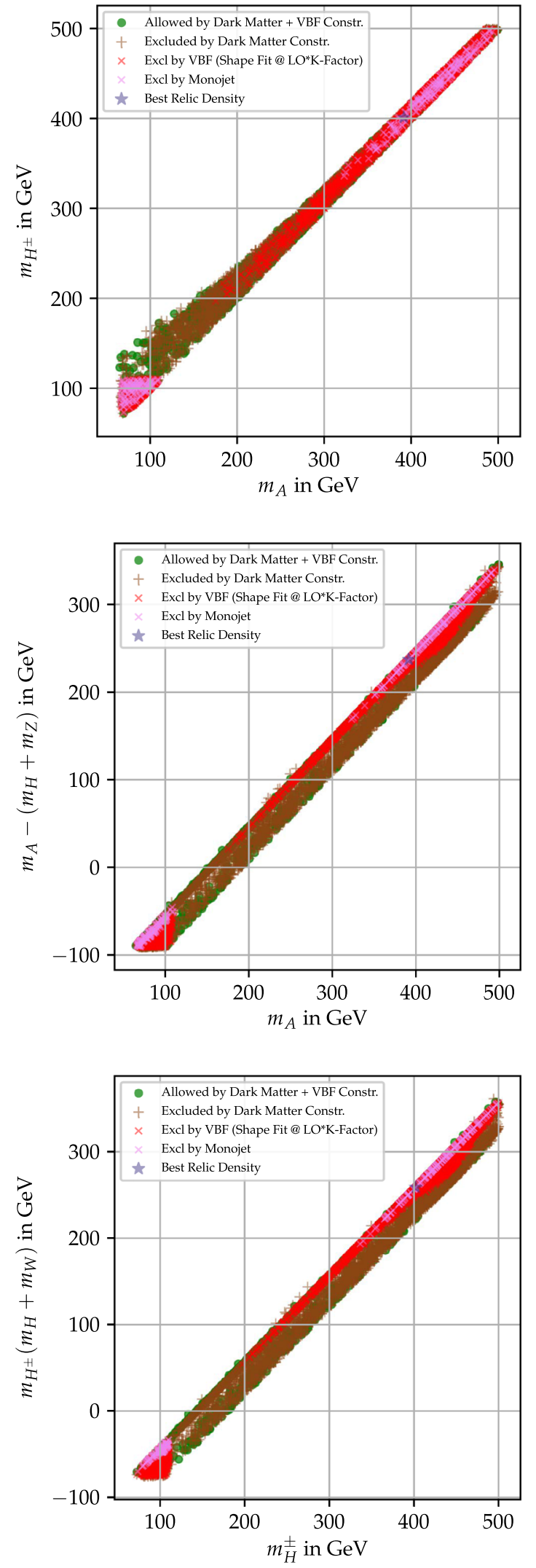

Fig. 5 Allowed combinations of the scalar masses $m_{H^{ \pm}}, m_{A}$ and $m_{H}$ which are relevant for collider analysis of the channels $p p \rightarrow H A$ and $p p \rightarrow H^{ \pm} A$ 
a dedicated Monte Carlo recast procedure is necessary in order to identify which points are subject to constraints from direct LHC searches.

Fortunately, CheckMATE bears the great advantage of being capable of quickly testing many such analyses simultaneously. We hence used it to perform a more inclusive scan of other potentially relevant final states. To be more precise, we considered the two body final states

$p p \rightarrow H A, H H^{ \pm}, A A, A H^{ \pm}$and $H^{+} H^{-}$.

We simulated all above processes in MG5_aMC@NLO, including a full consideration of the 2- and 3-body decays of $A$ and $H^{ \pm}$into $H$ and a set of Standard Model particles. These events are subsequently tested against all $13 \mathrm{TeV}$ analyses implemented in CheckMATE - a list is given in Appendix B.

It turns out that none of our $>10,000$ considered parameter tuples appear to be excluded by any search other than the already considered VBF and monojet channels.

Note that though we inclusively test all possible final states, the highest sensitivity is expected from leptonic final states, i.e.

$$
\begin{aligned}
& p p \rightarrow A H, A \rightarrow Z_{\text {lep }}^{(*)} H \\
& p p \rightarrow A H^{ \pm}, A \rightarrow Z_{\text {lep }}^{(*)} H, H^{ \pm} \rightarrow W_{\text {lep }}^{ \pm} H .
\end{aligned}
$$

The first signature is covered by Ref. [110] which searches for final state with invisible particles produced in association with a leptonically decaying $Z$-boson. ${ }^{16}$ We refer to this analysis as " $2 \ell$ " in the following. In contrast, the second example signature is covered ${ }^{17}$ by Ref. [111] - for short " $3 \ell$ " in the following text - which looks for various leptonic (and hadronic) final states in supersymmetric electroweakino production, i.e. $\tilde{\chi}_{1}^{+} \tilde{\chi}_{1}^{-}$and $\tilde{\chi}_{1}^{ \pm} \tilde{\chi}_{2}^{0}$. The expected final state for mixed chargino-neutralino production is experimentally identical to the aforementioned $A H^{ \pm}$decay chain and thus may be used to constrain the IDM.

To understand the reason for the non-sensitivity of current electroweakino searches, we show our results for the $A H$ channel and the $H^{ \pm} A$ channel in Fig. 6. In each subplot, we show the respective $r$-value of the analysis, defined as the ratio of the signal predicted by CheckMATE for the most sensitive signal region and the model-independent upper limit on

\footnotetext{
16 Note that this final state has been analysed before in Ref. [20] using Run 1 dilepton final states. However, the parameter regions they consider are excluded after applying constraints from dark matter relic density and the invisible width of the SM Higgs boson.

17 Note that Checkmate, and therefore also our analysis, makes use of preliminary results in Ref. [111] which were subsequently updated by a full publication in Ref. [112]. However, the published results are identical to those in the preliminary conference note.
}
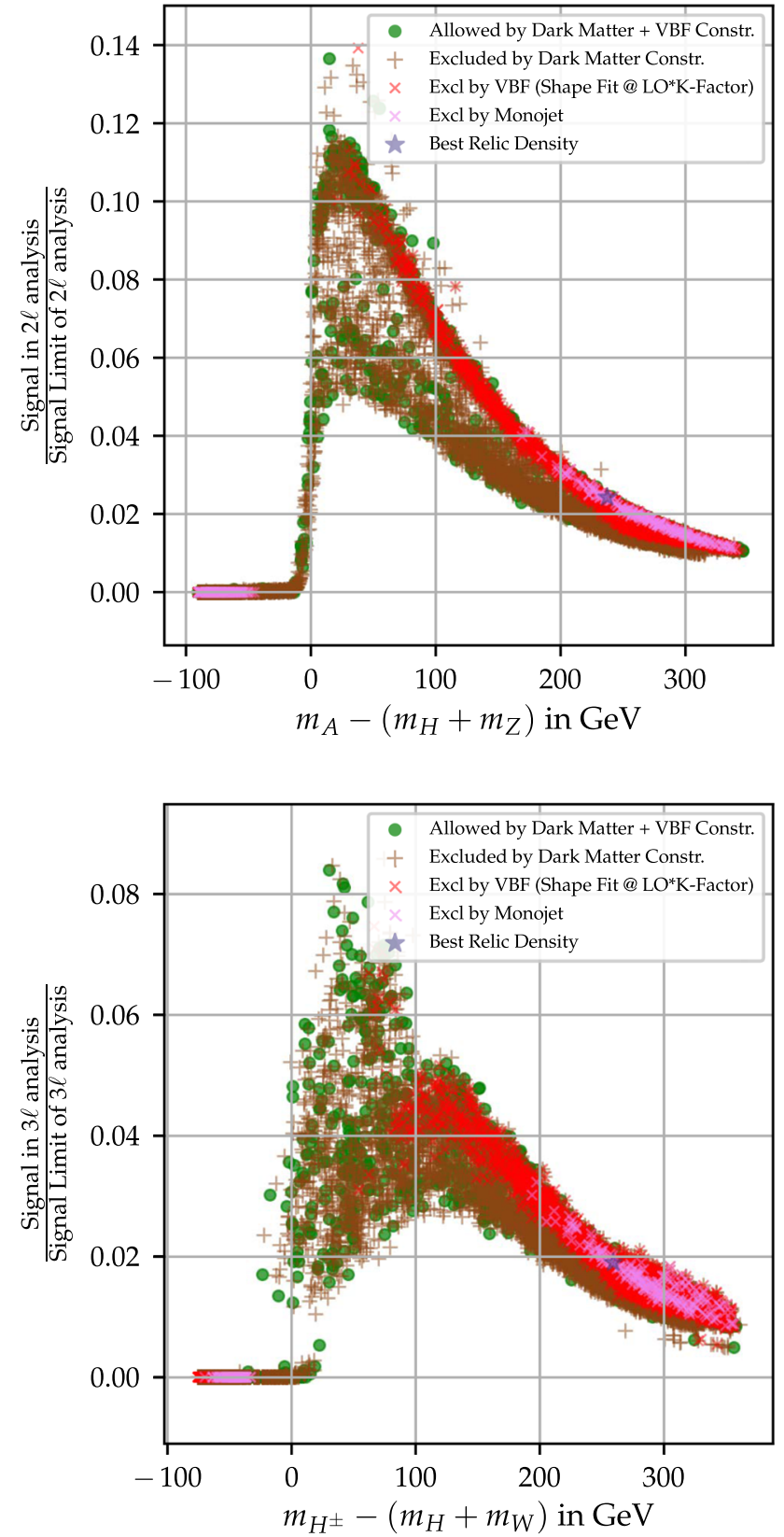

Fig. 6 Results for our LHC reinterpretation of SUSY electroweakino results on the masses of the IDM. The $x$-axis shows mass differences which are strongly correlated to the MET distribution in the final state. The $y$-axis denotes the $r$-value, defined as the ratio of the signal prediction divided by the $95 \%$ confidence limit on the signal

a signal in this signal region. Most importantly, $r$ scales with the predicted signal cross section and a value of $r \geq 1$ can be interpreted as a model point excluded at $95 \%$ confidence level.

For the $x$-axis, we respectively show the mass difference of a heavy inert scalar, $A$ or $H^{ \pm}$, and the summed masses of the two particles it decays into, e.g. $m_{A}-\left(m_{Z}+m_{H}\right)$ for $A \rightarrow Z H$. As explained before, the mass difference provides 
an estimate for the typical energy given to the leptons and to the dark matter candidate $H$ in the form of MET.

As can be seen from the figures, there is no $1: 1$ correspondence between the aforementioned mass difference and the model exclusion. This is obvious since the limit also depends on the absolute mass scales which for a given mass difference can change within $\pm 50 \mathrm{GeV}$, c.f. Fig. 5. However, one observes an overall rise-and-fall of the sensitivity and a global maximum near $m_{A}-\left(m_{H}+m_{Z}\right) \approx 50 \mathrm{GeV}$ and $m_{H^{ \pm}}-\left(m_{H}+m_{W^{ \pm}}\right) \approx 125 \mathrm{GeV}$. This structure can be explained from our discussion at the beginning of this section: The larger the mass difference, the higher the expected amount of lepton $p_{T}$ and MET in the final state becomes and so the overall signal efficiency increases. However, in order to obtain higher mass differences, electroweak precision constraints require larger masses for $A$ and $H^{ \pm}$and thus generally predicts smaller cross sections for viable IDM realisations. Hence, large mass differences simultaneously increase the final state efficiency and decrease the expected cross section. For the $2 \ell$ analysis, this results in a peak at a mass difference of approximately $30 \mathrm{GeV}$ which is related to the minimum $p_{T}$ cut on the signal leptons and the MET requirement of this analysis. For the $3 \ell$ analysis, no overall peak can be determined as the final state consists of two separate decay chains whose kinematic configurations simultaneously depend on $m_{A}$ and $m_{H^{ \pm}}$. Still, a similar behaviour can be observed.

However, as can be seen, the peak values for both analyses still only predict at most $15 \%$ of the required number of events for the analyses to be sensitive to the signal. Hence, we conclude that electroweakino searches are currently not sensitive to the IDM and from a statistical point of view, this may only change in the high luminosity limit of LHC 14. Still, the presented analysis only shows reinterpreted results motivated from different signal models and therefore not necessarily optimised towards the IDM. It may therefore be possible that a collider search specifically targeting the IDM may improve upon the results determined here via reinterpretation.

As an example, Fig. 7 illustrates how many events with leptonic final states are respectively expected from the processes in Eqs. (25), (26), without applying any event selection cuts. Note that for a signal to be observable e.g. in the $2 \ell$ analyses one requires at least 200 events after requiring the missing transverse momentum to be at least $90 \mathrm{GeV}$. It becomes apparent from Fig. 7 that a considerably softer cut on MET would significantly increase the number of expected signal events after cuts within the IDM. A full sensitivity study would however require the re-evaluation of SM background after modifying cuts. Such an analysis would provide important complementary information since, as can be seen from the different categories shown in Fig. 6, many points in
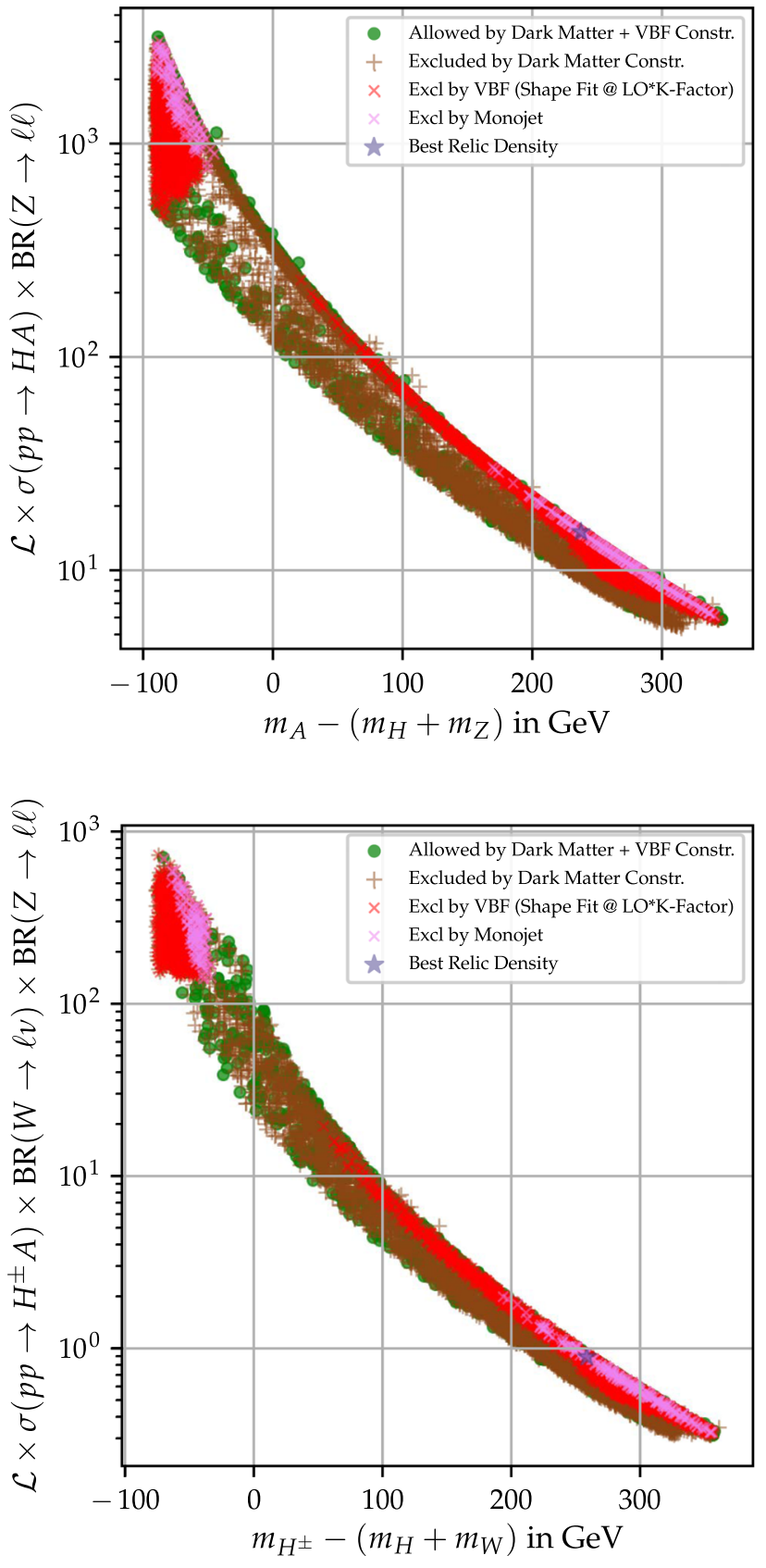

Fig. 7 Predicted number of events in the $2 \ell / 3 \ell$ channels. The $x$-axis shows mass differences as in Fig. 6. The $y$-axis denotes the product of integrated luminosity, total production cross section and leptonic branching ratio of the expected gauge boson(s) in the final state

the peak region of the direct search are neither excluded by the VBF channel nor by dark matter direct detection.

\section{Conclusions}

In this paper, we have considered the Inert Doublet Model, a two Higgs doublet model with a discrete $Z_{2}$ symmetry containing a scalar dark matter candidate. We have included all 
current theoretical and experimental collider constraints on this model as discussed in Ref. [45]. Concentrating on the region where $m_{H} \leq 100 \mathrm{GeV}$, we have investigated limits on the models' parameter space from a recast of recent LHC search where the invisibly decaying SM Higgs is produced either in vector boson fusion, Ref. [41], or in association with a hard jet, Ref. [42]. For this, we have implemented the above searches in the collider phenomenology tool CheckMATE and tested their sensitivity compared to constraints from dark matter and direct detection.

We observe that the VBF channel outperforms the monojet analysis and is sensitive to a large fraction of IDM parameter space and a proper recast of this analysis results in important bounds on the IDM model. Our search can significantly constrain a specific window in parameter space with dark matter masses $\sim 62-63 \mathrm{GeV}$ which evades dark matter limits due to an enhanced annihilation rate and leads to a significantly reduced relic abundance. This softens constraints from direct detection experiments like XENON1T. For larger masses, the VBF channel still provides relevant bounds which however do not improve direct detection limits within the pure IDM. The latter, however, could be avoided by coupling the lightest IDM scalar to a lighter dark matter sector which would have nearly no consequence for our presented collider analysis.

As no direct search for IDM scalars exist, we further reinterpret searches for BSM particles with the same experimental signature and conclude that these do not put further constraints on the IDM. We trace this back to the effect that either the cross section is too small or the mass splitting is not large enough to predict sufficiently high-energetic final state particles. In this context, it might be interesting to pursue whether a dedicated search for the inert scalars could enhance the expected LHC sensitivity and eventually provide complementary information to the VBF channel and dark matter direct detection, especially about the other scalar masses of the dark sector.

Acknowledgements This research was supported in parts by the National Science Centre, Poland, the HARMONIA project under contract UMO-2015/18/M/ST2/00518 (2016-2019), the National Science Foundation under Grant No. 1519045, by Michigan State University through computational resources provided by the Institute for CyberEnabled Research, by grant K 125105 of the National Research, Development and Innovation Fund in Hungary, and by the European Union through the European Regional Development Fund - the Competitiveness and Cohesion Operational Programme (KK.01.1.1.06). TR also thanks the DESY Theory group for their hospitality while parts of this work were completed. DD acknowledges funding and support from the Collaborative Research Unit (SFB) 676 of the Deutsche Forschungsgemeinschaft (DFG), project B1.

Data Availability Statement This manuscript has no associated data or the data will not be deposited. [Authors' comment: The research discussed in this article makes use of experimental results that are available in the format given in the publications of the LHC experimental collaborations, as cited in the manuscript. No additional data was generated.]
Open Access This article is distributed under the terms of the Creative Commons Attribution 4.0 International License (http://creativecomm ons.org/licenses/by/4.0/), which permits unrestricted use, distribution, and reproduction in any medium, provided you give appropriate credit to the original author(s) and the source, provide a link to the Creative Commons license, and indicate if changes were made.

Funded by $\mathrm{SCOAP}^{3}$.

\section{A IDM Feynman rules and other relations}

The parameters $m_{22}^{2}, \lambda_{3}, \lambda_{4}, \lambda_{5}$ can be re-expressed in terms of our input parameters:

$$
\begin{aligned}
& m_{22}^{2}=\lambda_{345} v^{2}-2 m_{H}^{2}, \\
& \lambda_{3}=\lambda_{345}-\frac{2}{v^{2}}\left(m_{H}^{2}-m_{H^{ \pm}}^{2}\right), \\
& \lambda_{4}=\frac{m_{A}^{2}+m_{H}^{2}-2 m_{H^{ \pm}}^{2}}{v^{2}}, \\
& \lambda_{5}=\frac{m_{H}^{2}-m_{A}^{2}}{v^{2}} .
\end{aligned}
$$

For completeness, we list the relevant Feynman rules of the IDM scalars in Tables 3, 4 and 5, omitting Goldstone modes as we are working in the unitary gauge at tree level. Note that the second, inert doublet neither participates in

Table 3 Triple scalar vertices

Table 4 Quartic scalar vertices

\begin{tabular}{lc}
\hline Vertex & Coupling \\
\hline$h H H$ & $\lambda_{345} v$ \\
$h A A$ & $\bar{\lambda}_{345} v$ \\
$h h h$ & $3 \lambda_{1} v$ \\
$h H^{+} H^{-}$ & $\lambda_{3} v$ \\
\hline & \\
\hline Vertex & Coupling \\
\hline$h h h h$ & $3 \lambda_{1}$ \\
$H^{+} H^{+} H^{-} H^{-}$ & $2 \lambda_{2}$ \\
$H H A A$ & $\lambda_{2}$ \\
$H H H H$ & $3 \lambda_{2}$ \\
$A A A A$ & $3 \lambda_{2}$ \\
$H^{+} H^{-} A A$ & $\lambda_{2}$ \\
$H^{+} H^{-} H H$ & $\lambda_{2}$ \\
$h h H^{+} H^{-}$ & $\lambda_{3}$ \\
$h h H H$ & $\lambda_{345}$ \\
$h h A A$ & $\bar{\lambda}_{345}$ \\
\hline & \\
\hline Vertex & $\operatorname{Coupling}$ \\
\hline$H^{-} H^{+} \gamma$ & $i e$ \\
$H^{-} H^{+} Z$ & $i \frac{g}{2} \frac{\cos \left(2 \theta_{W}\right)}{\cos \theta_{W}}$ \\
$H H^{ \pm} W^{\mp}$ & $\mp i \frac{g}{2}$ \\
$A H^{\mp} W^{ \pm}$ & $-\frac{g}{2}$ \\
$H A Z$ & $-\frac{g}{2 \cos \theta_{W}}$ \\
\hline &
\end{tabular}


Table 6 Full list of all $\sqrt{s}=13$ TeV CheckMATE analyses used for this study. Entries in boldface are relevant for the model studied in this work and are discussed in the main text. The column labelled \#SR yields the number of signal regions. Entries for the integrated luminosities $L_{\text {int }}$ are given in $\mathrm{fb}^{-1}$

\begin{tabular}{|c|c|c|c|c|}
\hline CheckMATE identifier & Search designed for & \#SR & $L_{\text {int }}$ & Ref. \\
\hline atlas_1602_09058 & Supersymmetry in final states with jets and two SS leptons or 3 leptons & 4 & 3.2 & [114] \\
\hline atlas_1604_01306 & New phenomena in events with a photon and $\mathbb{E}_{T}$ & 1 & 3.2 & [115] \\
\hline atlas_1604_07773 & New phenomena in final states with an energetic jet and large $\mathbb{E}_{T}$ & 13 & 3.2 & [116] \\
\hline atlas_1605_03814 & $\tilde{q}$ and $\tilde{g}$ in final states with jets and $\mathbb{E}_{T}$ & 7 & 3.2 & [117] \\
\hline atlas_1605_04285 & Gluinos in events with an isolated lepton, jets and $\mathbb{E}_{T}$ & 7 & 3.3 & [118] \\
\hline atlas_1605_09318 & Pair production of $\tilde{g}$ decaying via $\tilde{t}$ or $\tilde{b}$ in events with $b$-jets and $\mathbb{E}_{T}$ & 8 & 3.3 & [119] \\
\hline atlas_1606_03903 & $\tilde{t}$ in final states with one isolated lepton, jets and $\mathbb{E}_{T}$ & 3 & 3.2 & [120] \\
\hline atlas_1609_01599 & Measurement of $t t V$ cross sections in multilepton final states & 9 & 3.2 & [121] \\
\hline atlas_conf_2015_082 & Supersymmetry in events with leptonically decaying $Z$, jets and $\mathbb{E}_{T}$ & 1 & 3.2 & [122] \\
\hline atlas_conf_2016_013 & Vector-like $t$ pairs or $4 t$ in final states with leptons and jets & 10 & 3.2 & [123] \\
\hline atlas_conf_2016_050 & $\tilde{t}$ in final states with one isolated lepton, jets and $\mathbb{E}_{T}$ & 5 & 13.3 & [124] \\
\hline atlas_conf_2016_054 & $\tilde{q}, \tilde{g}$ in events with an isolated lepton, jets and $\mathbb{E}_{T}$ & 10 & 14.8 & [125] \\
\hline atlas_conf_2016_076 & Direct $\tilde{t}$ pair production and DM production in final states with $2 \ell$ & 6 & 13.3 & [126] \\
\hline atlas_conf_2016_078 & Further searches for $\tilde{q}$ and $\tilde{g}$ in final states with jets and $\mathbb{E}_{T}$ & 13 & 13.3 & [127] \\
\hline atlas_conf_2016_096 & Supersymmetry in events with $2 \ell$ or $3 \ell$ and $\boldsymbol{E}_{T}$ & 8 & 13.3 & [128] \\
\hline atlas_conf_2017_022 & $\tilde{q}, \tilde{g}$ in final states with jets and $\mathbb{E}_{T}$ & 24 & 36.1 & [129] \\
\hline atlas_conf_2017_039 & Electroweakino production in final states with 2 or 3 leptons & 37 & 36.1 & [111] \\
\hline atlas_conf_2017_040 & Dark Matter or invisibly decaying $h$, produced in associated with a $Z$ & 2 & 36.1 & [130] \\
\hline atlas_conf_2017_060 & New phenomena in final states with an energetic jet and large $\mathbb{E}_{T}$ & 13 & 36.1 & [131] \\
\hline cms_pas_sus_15_011 & New physics in final states with an OSSF lepton pair, jets and $\mathbb{E}_{T}$ & 47 & 2.2 & [132] \\
\hline cms_pas_hig_17_023 & Search for invisible decays of $h$ produced through VBF & 10 & 36.1 & {$[41]$} \\
\hline
\end{tabular}

electroweak symmetry breaking nor in the generation of fermion masses. Hence, the couplings of the SM-like Higgs $h$ to electroweak gauge bosons as well as fermions are given by their SM values, see e.g. [113], with the convention $g_{h W_{\mu}^{+} W_{v}^{-}}=i e^{2} v / 2 s_{W^{2}} g_{\mu \nu}$.

\section{B List of applied CheckMATE analyses}

Table 6 gives the full list of used CheckMATE analyses with a centre-of-mass energy of $\sqrt{s}=13 \mathrm{TeV}$. The first column shows the CheckMATE idenitifer, the second the purpose for which the analysis was designed for. The last three columns show the number of signal regions in the corresponding analysis (marked \#SR), the integrated luminosity for that analysis and the reference to the publication or conference notes from the experimental collaborations. We mark all analyses discussed in our main discussion in boldface. Note that Checkmate regularly implements preliminary results published as conference notes by the experimental LHC collaborations and use the corresponding conf-note identifiers. Often, these are published at a later stage by the collaborations without any changes to analysis procedure or results. More details on the individual analyses can be found in their respective references and corresponding validation material, if not provided in this work, can be found on http:// checkmate.hepforge.org.

\section{References}

1. N.G. Deshpande, E. Ma, Pattern of symmetry breaking with two Higgs doublets. Phys. Rev. D 18, 2574 (1978)

2. Q.-H. Cao, E. Ma, G. Rajasekaran, Observing the dark scalar doublet and its impact on the standard-model Higgs boson at colliders. Phys. Rev. D 76, 095011 (2007). arXiv:0708.2939

3. R. Barbieri, L.J. Hall, V.S. Rychkov, Improved naturalness with a heavy Higgs: an alternative road to LHC physics. Phys. Rev. D 74, 015007 (2006). arXiv:hep-ph/0603188

4. L. Lopez Honorez, E. Nezri, J.F. Oliver, M.H.G. Tytgat, The inert doublet model: an archetype for dark matter. JCAP 0702, 028 (2007). arXiv:hep-ph/0612275

5. L. Lopez Honorez, C.E. Yaguna, The inert doublet model of dark matter revisited. JHEP 1009, 046 (2010). arXiv: 1003.3125

6. E.M. Dolle, S. Su, The inert dark matter. Phys. Rev. D 80, 055012 (2009). arXiv:0906.1609

7. D. Sokolowska, Dark matter data and quartic self-couplings in inert doublet model. Acta Phys. Polon. B 42, 2237 (2011). arXiv: 1112.2953

8. I.F. Ginzburg, K.A. Kanishev, M. Krawczyk, D. Sokolowska, Evolution of Universe to the present inert phase. Phys. Rev. D 82, 123533 (2010). arXiv:1009.4593

9. T. Hambye, M.H.G. Tytgat, Electroweak symmetry breaking induced by dark matter. Phys. Lett. B 659, 651-655 (2008). arXiv:0707.0633 
10. T.A. Chowdhury, M. Nemevsek, G. Senjanovic, Y. Zhang, Dark matter as the trigger of strong electroweak phase transition. JCAP 1202, 029 (2012). arXiv: 1110.5334

11. D. Borah, J.M. Cline, Inert doublet dark matter with strong electroweak phase transition. Phys. Rev. D 86, 055001 (2012). arXiv: 1204.4722

12. G. Gil, P. Chankowski, M. Krawczyk, Inert dark matter and strong electroweak phase transition. Phys. Lett. B 717, 396-402 (2012). arXiv: 1207.0084

13. N. Blinov, S. Profumo, T. Stefaniak, The electroweak phase transition in the inert doublet model. JCAP 1507(07), 028 (2015). arXiv: 1504.05949

14. B. Swiezewska, M. Krawczyk, Diphoton rate in the inert doublet model with a $125 \mathrm{GeV}$ Higgs boson. Phys. Rev. D 88(3), 035019 (2013). arXiv:1212.4100

15. M. Gustafsson, S. Rydbeck, L. Lopez-Honorez, E. Lundstrom, Status of the inert doublet model and the role of multileptons at the LHC. Phys. Rev. D 86, 075019 (2012). arXiv:1206.6316

16. A. Arhrib, R. Benbrik, N. Gaur, $H \rightarrow \gamma \gamma$ in inert Higgs doublet model. Phys. Rev. D 85, 095021 (2012). arXiv:1201.2644

17. A. Arhrib, Y.-L.S. Tsai, Q. Yuan, T.-C. Yuan, An updated analysis of inert Higgs doublet model in light of the recent results from LUX, PLANCK, AMS-02 and LHC. JCAP 1406, 030 (2014). arXiv: 1310.0358

18. M. Krawczyk, D. Sokolowska, P. Swaczyna, B. Swiezewska, Constraining inert dark matter by $R_{\gamma \gamma}$ and WMAP data. JHEP 1309, 055 (2013). arXiv: 1305.6266

19. A. Goudelis, B. Herrmann, O. Stal, Dark matter in the Inert Doublet Model after the discovery of a Higgs-like boson at the LHC. JHEP 1309, 106 (2013). arXiv:1303.3010

20. G. Belanger, B. Dumont, A. Goudelis, B. Herrmann, S. Kraml, D. Sengupta, Dilepton constraints in the Inert Doublet Model from Run 1 of the LHC. Phys. Rev. D 91(11), 115011 (2015). arXiv: 1503.07367

21. N. Blinov, J. Kozaczuk, D.E. Morrissey, A. de la Puente, Compressing the Inert Doublet Model. Phys. Rev. D 93(3), 035020 (2016). arXiv: 1510.08069

22. A. Ilnicka, M. Krawczyk, T. Robens, Inert Doublet Model in light of LHC Run I and astrophysical data. Phys. Rev. D 93(5), 055026 (2016). arXiv: 1508.01671

23. A. Ilnicka, T. Robens, T. Stefaniak, Constraining extended scalar sectors at the LHC and beyond. Mod. Phys. Lett. A 33(10n11), 1830007 (2018). arXiv: 1803.03594

24. A.D. Banik, D. Majumdar, Inert doublet dark matter with an additional scalar singlet and $125 \mathrm{GeV}$ Higgs boson. Eur. Phys. J. C 74(11), 3142 (2014). arXiv: 1404.5840

25. A.D. Banik, D. Majumdar, Low energy gamma ray excess confronting a singlet scalar extended inert doublet dark matter model. Phys. Lett. B 743, 420-427 (2015). arXiv:1408.5795

26. C. Bonilla, D. Sokolowska, N. Darvishi, J.L. Diaz-Cruz, M. Krawczyk, IDMS: inert dark matter model with a complex singlet. J. Phys. G 43(6), 065001 (2016). arXiv:1412.8730

27. A.D. Plascencia, Classical scale invariance in the inert doublet model. JHEP 09, 026 (2015). arXiv:1507.04996

28. E. Dolle, X. Miao, S. Su, B. Thomas, Dilepton signals in the inert doublet model. Phys. Rev. D 81, 035003 (2010). arXiv:0909.3094

29. P. Swaczyna, MSc thesis, University of Warsaw (2013)

30. A. Datta, N. Ganguly, N. Khan, S. Rakshit, Exploring collider signatures of the inert Higgs doublet model. Phys. Rev. D 95(1), 015017 (2017). arXiv:1610.00648

31. N. Wan, N. Li, B. Zhang, H. Yang, M.-F. Zhao, M. Song, G. Li, J.-Y. Guo, Searches for dark matter via mono-W production in inert doublet model at the LHC. Commun. Theor. Phys. 69(5), 617 (2018)

32. A. Belyaev, T.R.F. Perez Tomei, P.G. Mercadante, C.S. Moon, S. Moretti, S.F. Novaes, L. Panizzi, F. Rojas, M. Thomas, Advanc- ing LHC probes of dark matter from the inert two-Higgs-doublet model with the monojet signal. Phys. Rev. D 99(1), 015011 (2019). arXiv: 1809.00933

33. P. Poulose, S. Sahoo, K. Sridhar, Exploring the Inert Doublet Model through the dijet plus missing transverse energy channel at the LHC. Phys. Lett. B 765, 300-306 (2017). arXiv:1604.03045

34. M. Kadastik, K. Kannike, A. Racioppi, M. Raidal, Implications of the $125 \mathrm{GeV}$ Higgs boson for scalar dark matter and for the CMSSM phenomenology. JHEP 1205, 061 (2012). arXiv: 1112.3647

35. B. Swiezewska, Inert scalars and vacuum metastability around the electroweak scale. JHEP 07, 118 (2015). arXiv:1503.07078

36. N. Khan, S. Rakshit, Constraints on inert dark matter from the metastability of the electroweak vacuum. Phys. Rev. D 92, 055006 (2015). arXiv: 1503.03085

37. G.M. Pruna, T. Robens, Higgs singlet extension parameter space in the light of the LHC discovery. Phys. Rev. D 88(11), 115012 (2013). arXiv:1303.1150

38. T. Robens, T. Stefaniak, Status of the Higgs singlet extension of the standard model after LHC Run 1. Eur. Phys. J. C75(3), 104 (2015). arXiv: 1501.02234

39. D. de Florian et al., Handbook of LHC Higgs Cross Sections: 4. Deciphering the Nature of the Higgs Sector (2016). arXiv: 1610.07922

40. M. Espirito Santo, K. Hultqvist, P. Johansson, A. Lipniacka, Search for neutralino pair production at $\mathrm{s}^{* *}(1 / 2)$ from $192-\mathrm{GeV}$ to $208-\mathrm{GeV}$ (2003)

41. A.M. Sirunyan et al., Search for invisible decays of a Higgs boson produced through vector boson fusion in proton-proton collisions at $\sqrt{s}=13$ TeV. Phys. Lett. B 793, 520-551 (2019). arXiv: 1809.05937

42. Search for dark matter and other new phenomena in events with an energetic jet and large missing transverse momentum using the ATLAS detector. Technical Report ATLAS-CONF-2017-060, CERN, Geneva (2017)

43. M. Drees, H. Dreiner, D. Schmeier, J. Tattersall, J.S. Kim, CheckMATE: confronting your favourite new physics model with LHC Data. Comput. Phys. Commun. 187, 227-265 (2014). arXiv: 1312.2591

44. D. Dercks, N. Desai, J.S. Kim, K. Rolbiecki, J. Tattersall, T. Weber, CheckMATE 2: from the model to the limit. Comput. Phys. Commun. 221, 383-418 (2017). arXiv:1611.09856

45. J. Kalinowski, W. Kotlarski, T. Robens, D. Sokolowska, A.F. Zarnecki, Benchmarking the Inert Doublet Model for $e^{+} e^{-}$colliders. JHEP 12, 081 (2018). arXiv: 1809.07712

46. L. Chuzhoy, E.W. Kolb, Reopening the window on charged dark matter. JCAP 0907, 014 (2009). arXiv:0809.0436

47. S. Kanemura, T. Kubota, E. Takasugi, Lee-Quigg-Thacker bounds for Higgs boson masses in a two doublet model. Phys. Lett. B 313, 155-160 (1993). arXiv:hep-ph/9303263

48. A.G. Akeroyd, A. Arhrib, E.-M. Naimi, Note on tree level unitarity in the general two Higgs doublet model. Phys. Lett. B 490, 119124 (2000). arXiv:hep-ph/0006035

49. M. Gustafsson, E. Lundstrom, L. Bergstrom, J. Edsjo, Significant gamma lines from inert Higgs dark matter. Phys. Rev. Lett. 99, 041301 (2007). arXiv:astro-ph/0703512

50. M.H.G. Tytgat, The inert doublet model: a new archetype of WIMP dark matter? J. Phys. Conf. Ser. 120, 042026 (2008). arXiv:0712.4206

51. P. Agrawal, E.M. Dolle, C.A. Krenke, Signals of inert doublet dark matter in neutrino telescopes. Phys. Rev. D 79, 015015 (2009). arXiv:0811.1798

52. E. Lundstrom, M. Gustafsson, J. Edsjo, The inert doublet model and LEP II limits. Phys. Rev. D 79, 035013 (2009). arXiv:0810.3924 
53. C. Arina, F.-S. Ling, M.H.G. Tytgat, IDM and iDM or the inert doublet model and inelastic dark matter. JCAP 0910, 018 (2009). arXiv:0907.0430

54. M. Krawczyk, D. Sokolowska, Constraining the Dark 2HDM. In 21st Rencontres de Blois on Windows on the Universe Blois, France, June 21-27, 2009 (2009). arXiv:0911.2457

55. M. Gustafsson, The Inert Doublet Model and Its Phenomenology. PoS, CHARGED2010:030 (2010). arXiv:1106.1719

56. B. Swiezewska, Yukawa independent constraints for two-Higgsdoublet models with a $125 \mathrm{GeV}$ Higgs boson. Phys. Rev. D 88(5), 055027 (2013). arXiv: 1209.5725

57. K.P. Modak, D. Majumdar, Confronting galactic and extragalactic $\gamma$-rays observed by Fermi-lat with annihilating dark matter in an inert Higgs doublet model. Astrophys. J. Suppl. 219(2), 37 (2015). 1502.05682

58. M. Hashemi, M. Krawczyk, S. Najjari, A.F. Zarnecki, Production of inert scalars at the high energy $e^{+} e^{-}$colliders (2015). arXiv:1512.01175 [JHEP 02, 187 (2016)]

59. M.A. Díaz, B. Koch, S. Urrutia-Quiroga, Constraints to dark matter from inert Higgs doublet model. Adv. High Energy Phys. 2016, 8278375 (2016). arXiv: 1511.04429

60. P.M. Ferreira, B. Swiezewska, One-loop contributions to neutral minima in the inert doublet model. JHEP 04, 099 (2016). arXiv: 1511.02879

61. R. Longas, D. Portillo, D. Restrepo, O. Zapata, The Inert Zee Model. JHEP 03, 162 (2016). arXiv: 1511.01873

62. M. Hashemi, S. Najjari, Observability of inert scalars at the LHC. Eur. Phys. J. C 77(9), 592 (2017). arXiv:1611.07827

63. A. Alves, D.A. Camargo, A.G. Dias, R. Longas, C.C. Nishi, F.S. Queiroz, Collider and dark matter searches in the inert doublet model from Peccei-Quinn symmetry. JHEP 10, 015 (2016). arXiv: 1606.07086

64. S. Kanemura, M. Kikuchi, K. Sakurai, Testing the dark matter scenario in the inert doublet model by future precision measurements of the Higgs boson couplings. Phys. Rev. D 94(11), 115011 (2016). arXiv: 1605.08520

65. D. Borah, A. Gupta, New viable region of an inert Higgs doublet dark matter model with scotogenic extension. Phys. Rev. D 96(11), 115012 (2017). arXiv: 1706.05034

66. B. Eiteneuer, A. Goudelis, J. Heisig, The inert doublet model in the light of Fermi-LAT gamma-ray data: a global fit analysis. Eur. Phys. J. C 77(9), 624 (2017). arXiv:1705.01458

67. J. Heisig, S. Kraml, A. Lessa, Constraining new physics with searches for long-lived particles: implementation into SModelS. Phys. Lett. B 788, 87-95 (2019). arXiv:1808.05229

68. D. Eriksson, J. Rathsman, O. Stal, 2HDMC: two-Higgs-doublet model calculator. Comput. Phys. Commun. 181, 833-834 (2010)

69. I.F. Ginzburg, K.A. Kanishev, Different vacua in 2HDM. Phys. Rev. D 76, 095013 (2007). arXiv:0704.3664

70. D. Sokolowska, Temperature evolution of physical parameters in the Inert Doublet Model. High Energy Phys. 1104, 3326 (2011)

71. A. Castillo, R.A. Diaz, J. Morales, C.G. Tarazona, Study of vacuum behavior for inert models with discrete $Z_{2}$-like and abelian $U(1)$ symmetries (2015). arXiv: 1510.00494

72. M. Lindner, M. Platscher, C.E. Yaguna, A. Merle, Fermionic WIMPs and vacuum stability in the scotogenic model. Phys. Rev. D 94(11), 115027 (2016). arXiv: 1608.00577

73. G. Aad et al., Combined measurement of the Higgs boson mass in $p p$ Collisions at $\sqrt{s}=7$ and $8 \mathrm{TeV}$ with the ATLAS and CMS experiments. Phys. Rev. Lett. 114, 191803 (2015). arXiv: 1503.07589

74. Measurements of Higgs boson properties from on-shell and offshell production in the four-lepton final state. Technical Report CMS-PAS-HIG-18-002, CERN, Geneva (2018)

75. K.A. Olive et al., Review of particle physics. Chin. Phys. C38, 090001 (2014)
76. G. Altarelli, R. Barbieri, Vacuum polarization effects of new physics on electroweak processes. Phys. Lett. B 253, 161 (1991)

77. M.E. Peskin, T. Takeuchi, A new constraint on a strongly interacting Higgs sector. Phys. Rev. Lett. 65, 964-967 (1990)

78. M.E. Peskin, T. Takeuchi, Estimation of oblique electroweak corrections. Phys. Rev. D 46, 381-409 (1992)

79. I. Maksymyk, C.P. Burgess, D. London, Beyond S, T and U. Phys. Rev. D 50, 529-535 (1994). arXiv:hep-ph/9306267

80. A. Pierce, J. Thaler, Natural dark matter from an unnatural Higgs boson and new colored particles at the TeV Scale. JHEP 0708, 026 (2007). arXiv:hep-ph/0703056

81. P. Bechtle, O. Brein, S. Heinemeyer, G. Weiglein, K.E. Williams, Higgs bounds: confronting arbitrary Higgs sectors with exclusion bounds from LEP and the tevatron. Comput. Phys. Commun. 181, 138-167 (2010). arXiv:0811.4169

82. P. Bechtle, O. Brein, S. Heinemeyer, G. Weiglein, K.E. Williams, HiggsBounds 2.0.0: confronting neutral and charged Higgs sector predictions with exclusion bounds from LEP and the tevatron. Comput. Phys. Commun. 182, 2605-2631 (2011). arXiv: 1102.1898

83. P. Bechtle, O. Brein, S. Heinemeyer, O. Stal, T. Stefaniak et al., HiggsBounds - 4: improved tests of extended Higgs sectors against exclusion bounds from LEP, the tevatron and the LHC. Eur. Phys. J. C 74(3), 2693 (2014). arXiv:1311.0055

84. P. Bechtle, S. Heinemeyer, O. Stal, T. Stefaniak, G. Weiglein, Applying exclusion likelihoods from LHC searches to extended Higgs sectors. Eur. Phys. J. C 75(9), 421 (2015). arXiv:1507.06706

85. http://higgsbounds.hepforge.org

86. V. Khachatryan et al., Searches for invisible decays of the Higgs boson in pp collisions at sqrt(s) $=7,8$, and $13 \mathrm{TeV}$. JHEP 02, 135 (2017). arXiv: 1610.09218

87. G. Aad et al., Measurements of the Higgs boson production and decay rates and constraints on its couplings from a combined ATLAS and CMS analysis of the LHC pp collision data at $\sqrt{s}=7$ and 8 TeV. JHEP 08, 045 (2016). arXiv: 1606.02266

88. P. Bechtle, S. Heinemeyer, O. Stal, T. Stefaniak, G. Weiglein, Higgs Signals: confronting arbitrary Higgs sectors with measurements at the Tevatron and the LHC. Eur. Phys. J. C 74(2), 2711 (2014). arXiv:1305.1933

89. N. Aghanim et al., Planck 2018 results. VI. Cosmological parameters (2018). arXiv: 1807:06209

90. C. Cheung, L.J. Hall, D. Pinner, J.T. Ruderman, Prospects and blind spots for neutralino dark matter. JHEP 05, 100 (2013). arXiv: 1211.4873

91. D. Barducci, G. Belanger, J. Bernon, F. Boudjema, J. Da Silva, S. Kraml, U. Laa, A. Pukhov, Collider limits on new physics within micrOMEGAs_4.3. Comput. Phys. Commun. 222, 327338 (2018). arXiv:1606.03834

92. E. Aprile et al., Dark matter search results from a one ton-year exposure of XENON1T. Phys. Rev. Lett. 121(11), 111302 (2018). arXiv: 1805.12562

93. A. Belyaev, J. Blandford, D. Locke, Phenodata database (2017). https://hepmdb.soton.ac.uk/phenodata

94. Q.-H. Cao, E. Ma, J. Wudka, C.P. Yuan, Multipartite dark matter (2007). arXiv:0711.3881

95. G. Bélanger, K. Kannike, A. Pukhov, M. Raidal, Minimal semiannihilating $\mathbb{Z}_{N}$ scalar dark matter. JCAP 1406, 021 (2014). arXiv: 1403.4960

96. G. Bélanger, F. Boudjema, A. Pukhov, A. Semenov, micrOMEGAs4.1: two dark matter candidates. Comput. Phys. Commun. 192, 322-329 (2015). 1407.6129

97. M. Badziak, A. Delgado, M. Olechowski, S. Pokorski, K. Sakurai, Detecting underabundant neutralinos. JHEP 11, 053 (2015). arXiv: 1506.07177 
98. A. Belyaev, G. Cacciapaglia, I.P. Ivanov, F. Rojas-Abatte, M. Thomas, Anatomy of the inert two Higgs doublet model in the light of the LHC and non-LHC dark matter searches. Phys. Rev. D 97(3), 035011 (2018). arXiv:1612.00511

99. J.S. Kim, D. Schmeier, J. Tattersall, K. Rolbiecki, A framework to create customised LHC analyses within CheckMATE. Comput. Phys. Commun. 196, 535-562 (2015). arXiv: 1503.01123

100. P. Nason, A new method for combining NLO QCD with shower Monte Carlo algorithms. JHEP 11, 040 (2004). arXiv:hep-ph/0409146

101. S. Frixione, P. Nason, C. Oleari, Matching NLO QCD computations with Parton Shower simulations: the POWHEG method. JHEP 11, 070 (2007). arXiv:0709.2092

102. P. Nason, C. Oleari, NLO Higgs boson production via vectorboson fusion matched with shower in POWHEG. JHEP 02, 037 (2010). arXiv:0911.5299

103. S. Alioli, P. Nason, C. Oleari, E. Re, A general framework for implementing NLO calculations in shower Monte Carlo programs: the POWHEG BOX. JHEP 06, 043 (2010). arXiv: 1002.2581

104. T. Sjostrand, S. Mrenna, P.Z. Skands, PYTHIA 6.4 Physics and Manual. JHEP 05, 026 (2006). arXiv:hep-ph/0603175

105. J. Alwall, M. Herquet, F. Maltoni, O. Mattelaer, T. Stelzer, MadGraph 5: going beyond. JHEP 1106, 128 (2011). arXiv:1106.0522

106. T. Sjoestrand, S. Ask, J.R. Christiansen, R. Corke, N. Desai, P. Ilten, S. Mrenna, S. Prestel, C.O. Rasmussen, P.Z. Skands, An introduction to PYTHIA 8.2. Comput. Phys. Commun. 191, 159177 (2015). arXiv: 1410.3012

107. B. Dutta, G. Palacio, J.D. Ruiz-Alvarez, D. Restrepo, Vector boson fusion in the inert doublet model. Phys. Rev. D 97(5), 055045 (2018). arXiv: 1709.09796

108. D.S. Akerib et al., First results from the LUX dark matter experiment at the Sanford Underground Research Facility. Phys. Rev. Lett. 112(9), 091303 (2014). arXiv:1310.8214

109. A. Ibarra, C.E. Yaguna, O. Zapata, Direct detection of fermion dark matter in the radiative seesaw model. Phys. Rev. D 93(3), 035012 (2016). arXiv: 1601.01163

110. M. Aaboud et al., Search for an invisibly decaying Higgs boson or dark matter candidates produced in association with a $Z$ boson in $p p$ collisions at $\sqrt{s}=13 \mathrm{TeV}$ with the ATLAS detector. Phys. Lett. B 776, 318-337 (2018). arXiv:1708.09624

111. Search for electroweak production of supersymmetric particles in the two and three lepton final state at $\sqrt{s}=13 \mathrm{TeV}$ with the ATLAS detector. Technical Report ATLAS-CONF-2017-039, CERN, Geneva (2017)

112. M. Aaboud et al., Search for electroweak production of supersymmetric particles in final states with two or three leptons at $\sqrt{s}=13 \mathrm{TeV}$ with the ATLAS detector. Eur. Phys. J. C 78(12), 995 (2018). arXiv: 1803.02762

113. M. Bohm, A. Denner, H. Joos, Gauge theories of the strong and electroweak interaction (2001)

114. G. Aad et al., Search for supersymmetry at $\sqrt{s}=13 \mathrm{TeV}$ in final states with jets and two same-sign leptons or three leptons with the ATLAS detector. Eur. Phys. J. C 76(5), 259 (2016). arXiv: 1602.09058

115. M. Aaboud et al., Search for new phenomena in events with a photon and missing transverse momentum in $p p$ collisions at $\sqrt{s}=13 \mathrm{TeV}$ with the ATLAS detector. JHEP 06, 059 (2016). arXiv: 1604.01306

116. M. Aaboud et al., Search for new phenomena in final states with an energetic jet and large missing transverse momentum in $p p$ collisions at $\sqrt{s}=13 \mathrm{TeV}$ using the ATLAS detector. Phys. Rev. D 94(3), 032005 (2016). arXiv:1604.07773

117. M. Aaboud et al., Search for squarks and gluinos in final states with jets and missing transverse momentum at $\sqrt{s}=13 \mathrm{TeV}$ with the ATLAS detector. Eur. Phys. J. C 76(7), 392 (2016). arXiv: 1605.03814

118. G. Aad et al., Search for gluinos in events with an isolated lepton, jets and missing transverse momentum at $\sqrt{s}=13 \mathrm{Te} V$ with the ATLAS detector. Eur. Phys. J. C 76(10), 565 (2016) arXiv: 1605.04285

119. G. Aad et al., Search for pair production of gluinos decaying via stop and sbottom in events with $b$-jets and large missing transverse momentum in $p p$ collisions at $\sqrt{s}=13 \mathrm{TeV}$ with the ATLAS detector. Phys. Rev. D 94(3), 032003 (2016). arXiv:1605.09318

120. M. Aaboud et al., Search for top squarks in final states with one isolated lepton, jets, and missing transverse momentum in $\sqrt{s}=$ $13 \mathrm{TeV} p p$ collisions with the ATLAS detector. Phys. Rev. D 94(5), 052009 (2016). arXiv:1606.03903

121. M. Aaboud et al., Measurement of the $t \bar{t} Z$ and $t \bar{t} W$ production cross sections in multilepton final states using $3.2 \mathrm{fb}^{-1}$ of $p p$ collisions at $\sqrt{s}=13 \mathrm{TeV}$ with the ATLAS detector. Eur. Phys. J. C 77(1), 40 (2017). arXiv:1609.01599

122. A search for Supersymmetry in events containing a leptonically decaying $Z$ boson, jets and missing transverse momentum in $\sqrt{s}=13 \mathrm{TeV} p p$ collisions with the ATLAS detector. Technical Report ATLAS-CONF-2015-082, CERN, Geneva (2015)

123. Search for production of vector-like top quark pairs and of four top quarks in the lepton-plus-jets final state in $p p$ collisions at $\sqrt{s}=13 \mathrm{TeV}$ with the ATLAS detector. Technical Report ATLAS-CONF-2016-013, CERN, Geneva (2016)

124. Search for top squarks in final states with one isolated lepton, jets, and missing transverse momentum in $\sqrt{s}=13 \mathrm{TeV}$ pp collisions with the ATLAS detector. Technical Report ATLAS-CONF-2016050, CERN, Geneva, (Aug 2016)

125. Search for squarks and gluinos in events with an isolated lepton, jets and missing transverse momentum at $\sqrt{s}=13 \mathrm{TeV}$ with the ATLAS detector. Technical Report ATLAS-CONF-2016-054, CERN, Geneva (2016)

126. Search for direct top squark pair production and dark matter production in final states with two leptons in $\sqrt{s}=13 \mathrm{TeV} p p$ collisions using $13.3 \mathrm{fb}^{-1}$ of ATLAS data. Technical Report ATLASCONF-2016-076, CERN, Geneva (2016)

127. Further searches for squarks and gluinos in final states with jets and missing transverse momentum at $\sqrt{s}=13 \mathrm{TeV}$ with the ATLAS detector. Technical Report ATLAS-CONF-2016-078, CERN, Geneva (2016)

128. Search for supersymmetry with two and three leptons and missing transverse momentum in the final state at $\sqrt{s}=13 \mathrm{TeV}$ with the ATLAS detector. Technical Report ATLAS-CONF-2016-096, CERN, Geneva (2016)

129. Search for squarks and gluinos in final states with jets and missing transverse momentum using $36 \mathrm{fb}^{1}$ of $\sqrt{s}=13 \mathrm{TeV}$ pp collision data with the ATLAS detector. Technical Report ATLAS-CONF2017-022, CERN, Geneva (2017)

130. Search for an invisibly decaying Higgs boson or dark matter candidates produced in association with a $Z$ boson in $p p$ collisions at $\sqrt{s}=13 \mathrm{TeV}$ with the ATLAS detector. Technical Report ATLAS-CONF-2017-040, CERN, Geneva (2017)

131. M. Aaboud et al., Search for dark matter and other new phenomena in events with an energetic jet and large missing transverse momentum using the ATLAS detector. JHEP 01, 126 (2018). arXiv: 1711.03301

132. Search for new physics in final states with two opposite-sign sameflavor leptons, jets and missing transverse momentum in pp collisions at $\operatorname{sqrt}(\mathrm{s})=13 \mathrm{TeV}$. Technical Report CMS-PAS-SUS-15011, CERN, Geneva (2015) 